\title{
Observers for a non-Lipschitz triangular form
}

\author{
P. Bernard ${ }^{a}$, L. Praly $^{a}$, V. Andrieu ${ }^{b}$ \\ ${ }^{a}$ Centre Automatique et Systèmes, MINES ParisTech, PSL Research University, France \\ ${ }^{\mathrm{b}}$ Université Lyon 1, Villeurbanne, France - CNRS, UMR 5007, LAGEP, France
}

\begin{abstract}
We address the problem of designing an observer for triangular non locally Lipschitz dynamical systems. We show the convergence with an arbitrary small error of the classical high gain observer in presence of nonlinearities verifying some Hölderlike condition. Also, for the case when this Hölder condition is not verified, we propose a novel cascaded high gain observer. Under slightly more restrictive assumptions, we prove the convergence of an homogeneous observer and of its cascaded version with the help of an explicit Lyapunov function.
\end{abstract}

Key words: triangular observable form, high-gain observer, finite-time observers, homogeneous observers, exact differentiators, explicit Lyapunov functions

\section{Introduction}

A preliminary step is often required in the construction of observers for controlled nonlinear systems. It consists in finding a reversible coordinate transformation, allowing us to rewrite the system dynamics in a target form more favorable for writing and/or analyzing the observer. For example, the dynamics of a controlled single output system of dimension $n$ which is uniformly observable (see [12, Definition I.2.1.2]) and differentially observable of order $m$ (see [12, Definition I.2.4.2]) with $m=n$ can be written with appropriate coordinates in a Lipschitz triangular form appropriate for the design of a high gain observer $(10,11)$. Such a property is no more true when the order $m$ is strictly larger than the dimension $n$. Indeed in this case, we may still get the usual triangular form but with functions that may not be Lipschitz ([6]). A particular case of this is when there is only one nonlinear function (in the last line for the single output case). This is the so-called phase-variable form. It has been known for a long time, in particular in the context of dirty-derivatives and output differentiation, that a high gain observer can provide an arbitrary small error as long as the nonlinearity is bounded ([26] among many others). We also know since [14 that a sliding mode observer can achieve finite-time convergence under the same assumption. In this paper, we want to build observers for the more general triangular canonical form where non-Lipschitz triangular nonlinearities can appear on any line. As far as we know, this form has not received much attention apart from its well-known

Email addresses: pauline.bernard@mines-paristech.fr (P. Bernard), laurent.praly@mines-paristech.fr (L. Praly), vincent.andrieu@gmail.com (V. Andrieu).
Lipschitz version and the convergence results holding for the phase-variable form do not extend trivially.

This paper follows and completes [5. We show here that the classical high gain observer may still be used when the nonlinearities verify some Hölder-type condition. Nevertheless, the asymptotic convergence is lost and only a convergence with an arbitrary small error remains. When the nonlinearities do not verify the required Hölder regularity, it is also possible to use a cascade of high gain observers, but once again, the convergence is only with an arbitrary small error.

Fortunately, moving to a generalization of high gain observer exploiting homogeneity makes it possible to achieve convergence. It is at the beginning of the century that researchers started to consider homogeneous observers with various motivations: exact differentiators (14, 15, 16]), domination as a tool for designing stabilizing output feedback (27, [20, 21], 3] and references therein (in particular 1 )), ... The advantage of this type of observers is their ability to face Hölder nonlinearities.

With the tools introduced in [2], we have at our disposal a Lyapunov design to obtain an homogeneous observer with degree in $]-1,0[$ for the triangular form mentioned above. By construction, convergence is guaranteed if the nonlinearities verify a Hölder-type condition. We show here that the same Lyapunov design can be extended to the case where the degree of homogeneity is -1 . This is interesting since the constraints on the nonlinearities become less and less restrictive when the degree gets closer to -1 . It turns out that, in the absence of nonlinearities, the observer we obtain is actually the exact differentiator presented in 14] and which is defined by an homogeneous differential inclusion. But as opposed to [14] 
where convergence is established via a solution-based analysis, in our case, convergence is again guaranteed by construction since the design gives also a homogeneous strict Lyapunov function. Moreover this function enables us to quantify the effect of the observer parameters on the behavior in presence of Hölder nonlinearities and disturbances. Of course, knowing the convergence of the exact differentiator from [14, we could have deduced the existence of such a Lyapunov function via a converse theorem as in 18. But with only existence, effect quantifications as mentioned above is nearly impossible. Actually many efforts have been made to get expressions for Lyapunov functions but, as opposed to Lyapunov design, Lyapunov analysis is much harder. As far as we know, expressions of Lyapunov functions have been obtained this way only for $m \leq 3$. See [19].

Finally, to face the unfortunate situation where the nonlinearities verify none of the above mentioned Hölder type conditions, we propose a novel observer made of a cascade of homogeneous observers whose maximal total dimension is $\frac{m(m+1)}{2}$. We prove that it converges without requiring anything on the nonlinearities (except continuity) in the case where the system trajectories and the input are bounded.

All along our paper, we sometimes use stronger assumptions than necessary in order to simplify the presentation of our results. We signal them to the reader with a symbol as in "the trajectories are complete ". We discuss how they can be relaxed later in Section 7 , in particular when we restrict our attention to compact sets.

\section{Notations}

(1) We define the signed power function as

$$
\lfloor a\rceil^{b}=\operatorname{sign}(a)|a|^{b},
$$

where $b$ is a nonnegative real number. In the particular case where $b=0,\lfloor a\rceil^{0}$ is actually any number in the set

$$
\mathrm{S}(a)= \begin{cases}\{1\} & \text { if } a>0 \\ {[-1,1]} & \text { if } a=0 \\ \{-1\} & \text { if } a<0\end{cases}
$$

Namely, writing $c=\lfloor a\rceil^{0}$ means $c \in \mathrm{S}(a)$. Note that the set valued map $a \mapsto S(a)$ is upper semicontinuous with nonempty, compact and convex values.

(2) For $\left(z_{1}, \ldots, z_{i}\right)$ and $\left(\hat{z}_{1}, \ldots, \hat{z}_{i}\right)\left(\operatorname{resp} .\left(\hat{z}_{i 1}, \ldots, \hat{z}_{i i}\right)\right)$ in $\mathbb{R}^{i}$, we denote

$$
\begin{gathered}
\mathbf{z}_{i}=\left(z_{1}, \ldots, z_{i}\right) \\
\hat{\mathbf{z}}_{i}=\left(\hat{z}_{1}, \ldots, \hat{z}_{i}\right) \quad\left(\operatorname{resp.} \quad \hat{\mathbf{z}}_{i}=\left(\hat{z}_{i 1}, \ldots, \hat{z}_{i i}\right)\right) \\
e_{i j}=\hat{z}_{i j}-z_{j}, \quad e_{j}=\hat{z}_{j}-z_{j}, \mathbf{e}_{i}=\hat{\mathbf{z}}_{i}-\mathbf{z}_{i} .
\end{gathered}
$$

\section{Continuous triangular form}

Consider a nonlinear system of the form

$$
\left\{\begin{array}{rl}
\dot{z}_{1} & =z_{2}+\Phi_{1}\left(u, z_{1}\right)+w_{1} \\
& \vdots \\
\dot{z}_{i} & =z_{i+1}+\Phi_{i}\left(u, z_{1}, \ldots, z_{i}\right)+w_{i}, \\
& \vdots \\
\dot{z}_{m} & =\Phi_{m}(u, z)+w_{m} \\
y & =z_{1}+v
\end{array},\right.
$$

where $z$ is the state in $\mathbb{R}^{m}, y$ is a measured output in $\mathbb{R}$, $\Phi$ is a continuous function which is not assumed to be locally Lipschitz. $w$ can model either a known or an unknown disturbance on the dynamics and $v$ is an unknown disturbance on the measurement. Given locally bounded measurable time functions $t \mapsto u(t)$ and $t \mapsto w(t)$, we denote $Z(z, t ; u, w)$ a solution of (2) going through $z$ at time 0 which, to simplify the presentation, is assumed to be defined for all $t \geq 0$ (i.e. the trajectories are complete ${ }^{\text {s }}$ ). We are interested in estimating $Z(z, t ; u, w)$ knowing $y$ and $u$.

As mentioned in the introduction, this kind of triangular continuous form, as we call (2), appears when we consider systems which are uniformly observable and differentially observable but with an order larger than the system's dimension. An example is given in Section 8.

The only existing observer we are aware of able to cope with $\Phi$ no more than continuous is the one presented in 4. Its dynamics are described by a differential inclusion (see Appendix A) :

$$
\dot{\hat{z}} \in F(\hat{z}, y, u)
$$

where $(\hat{z}, y, u) \mapsto F(\hat{z}, y, u)$ is a set valued map. In the disturbance free context (i.e. $v=w_{i}=0$ ), it can be shown that any absolutely continuous solution gives in finite time an estimate of $z$ under the only assumption of boundedness of the input and of the state trajectory. But the set valued map $F$ above does not satisfy the usual basic assumptions (upper semi-continuous with compact and convex values) (see 9, 22 ). It follows that we are not guaranteed of the existence of absolutely continuous solutions nor of possible sequential compactness of such solutions and therefore of possibilities of approximations of $F$. That is why, in this paper, we look for other candidate observers for the triangular form (2).

In doing so, we might have to restrict the possible nonlinearities allowed to obtain the existence of an observer. The restriction we will impose can be described as follows. For a positive real number $\mathfrak{a}$, and a vector $\alpha$ in $[0,1]^{\frac{m(m+1)}{2}}$, we will say that the function $\Phi$ verifies the property $\mathcal{P}(\alpha, \mathfrak{a})$ if :

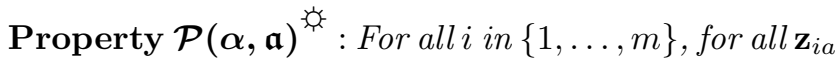


and $\mathbf{z}_{i b}$ in $\mathbb{R}^{m}$ and $u$ in $U$, we hav $£^{1}$ :

$$
\left|\Phi_{i}\left(u, \mathbf{z}_{i a}\right)-\Phi_{i}\left(u, \mathbf{z}_{i b}\right)\right| \leq \mathfrak{a} \sum_{j=1}^{i}\left|z_{j a}-z_{j b}\right|^{\alpha_{i j}}
$$

This property captures many possible contexts. In the case in which $\alpha_{i j}>0$, it implies that the function $\Phi$ is Hölder with power $\alpha_{i j}$. When the $\alpha_{i j}=0$, it simply implies that the function $\Phi$ is bounded. In the following, our aim is to design an observer depending on the values of $\alpha$.

It is possible to employ the degree of freedom given in (2) by the time functions $w$ to deal with the case in which the given function $\Phi(u, z)$ doesn't satisfy $\mathcal{P}(\mathfrak{a}, \alpha)$. In this case, an approximation procedure can be carried out to get a function $\hat{\Phi}$ satisfying $\mathcal{P}(\mathfrak{a}, \alpha)$ and selecting $w=\Phi(u, z)-\hat{\Phi}(u, z)$ which is an unknown disturbance. The quality of the estimates obtained from the observer will then depend on the quality of the approximation (ie the norm of $w$ ). This is what is done for example in 17 when dealing with locally Lipschitz approximations. We will further discuss in Section 7 how to relax assumption $\mathcal{P}(\mathfrak{a}, \alpha)$.

In Section 3, we start by showing the convergence with an arbitrary small error of the classical high gain observer when the nonlinearity $\Phi$ verifies the property $\mathcal{P}$ for certain values of $\alpha_{i j}$. We deduce in Section 4 the convergence with an arbitrary small error for a cascaded high gain observer when the input and the state trajectories are bounded. On an other hand, in Section 5, we show that replacing the high gain structure by an homogeneous structure enables to obtain convergence under a slightly more restrictive Hölder restriction. Then, a cascaded homogeneous observer is presented in Section 6, which ensures asymptotic convergence when the input and the state trajectories are bounded. As already mentioned, in Section 7, we indicate how the assumptions, marked with in the text, can be relaxed. Finally, we illustrate our observers with an example in Section 8.

\section{High gain observer}

We consider in this section a classical high gain observer:

$$
\left\{\begin{aligned}
\dot{\hat{z}}_{1} & =\hat{z}_{2}+\Phi_{1}\left(u, \hat{z}_{1}\right)+\hat{w}_{1}-L k_{1}\left(\hat{z}_{1}-y\right) \\
\dot{\hat{z}}_{2} & =\hat{z}_{3}+\Phi_{2}\left(u, \hat{z}_{1}, \hat{z}_{2}\right)+\hat{w}_{2}-L^{2} k_{2}\left(\hat{z}_{1}-y\right) \\
& \vdots \\
\dot{\hat{z}}_{m} & =\Phi_{m}(u, \hat{z})+\hat{w}_{m}-L^{m} k_{m}\left(\hat{z}_{1}-y\right)
\end{aligned}\right.
$$

where $L$ and the $k_{i}$ 's are gains to be tuned, $y$ is the measurement. The $\hat{w}_{i}$ are approximations of the $w_{i}$. In particular, when $w_{i}$ represents unknown disturbances,

1 Actually $\Phi_{i}$ can depend also on $z_{i+1}$ to $z_{m}$ as long as (3) holds. It can also depend on time requiring some uniform property (see Section 7). the corresponding $\hat{w}_{i}$ is simply taken equal to 0 . In the following, we denote

$$
\Delta w=\hat{w}-w
$$

When $\Phi$ satisfies the property $\mathcal{P}(\alpha, \mathfrak{a})$ with $\alpha_{i j}=1$ for all $1 \leq j \leq i \leq m$, we recognize the usual triangular Lipschitz property for which the nominal high-gain observer gives an input to state stability (ISS) property with respect to the measurement disturbance $v$ and dynamics disturbance $w$. It is well known that the ISS gain between the disturbance and the estimation error depends on the high-gain parameter $L$. Specifically, we have the following well known result. See for instance [13] for a proof.

Proposition 1 (Nominal high-gain) There exist real numbers $k_{1}, \ldots, k_{m}, L^{*}, \lambda, \beta$ and $\gamma$ such that,

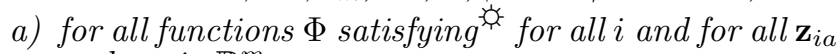
and $\mathbf{z}_{i b}$ in $\mathbb{R}^{m}$

$$
\left|\Phi_{i}\left(u, \mathbf{z}_{i a}\right)-\Phi_{i}\left(u, \mathbf{z}_{i b}\right)\right| \leq \mathfrak{a} \sum_{j=1}^{i}\left|z_{j a}-z_{j b}\right|+\mathfrak{b}_{i}
$$

b) for all $L \geq \max \left\{\mathfrak{a} L^{*}, 1\right\}$,

c) for all locally bounded time function $(u, v, w, \hat{w})$, all $(z, \hat{z})$ in $\mathbb{R}^{m} \times \mathbb{R}^{m}$,

any solution $\hat{Z}(\hat{z}, z, t ; u, v, w, \hat{w})$ of (4) verifies, for all $t_{0}$ and $t$ such that $t \geq t_{0} \geq 0$, and for all $i$ in $\{1, \ldots, m\}$,

$$
\begin{aligned}
& \left.\mid \hat{Z}_{i}(t)-Z_{i}(t)\right) \mid \\
& \leq \max \left\{L^{i-1} \beta \mid \hat{Z}_{i}\left(t_{0}\right)-Z_{i}\left(t_{0}\right)\right) \mid e^{-\lambda L\left(t-t_{0}\right)}, \\
& \left.\gamma \sup _{\substack{1 \leq j \leq m \\
s \in\left[t_{0}, t\right]}}\left\{L^{i-1}|v(s)|, \frac{\left|\Delta w_{j}(s)\right|+\mathfrak{b}_{j}}{L^{j-i+1}}\right\}\right\} .
\end{aligned}
$$

where we have used the abbreviation $Z(t)=Z(z, t ; u, w)$ and $\hat{Z}(t)=\hat{Z}(z, \hat{z}, t ; u, v, w, \hat{w})$.

Since the nominal high-gain observer gives asymptotic convergence for Lipschitz nonlinearities, we may wonder what type of property is preserved when the nonlinearities are only Hölder. In the following proposition, we show that the usual high-gain observer can provide an arbitrary small error on the estimate providing the Hölder orders $\alpha_{i j}$ satisfy the restrictions given in Table 1 or Equation (7).

Proposition 2 Assume the function $\Phi$ verifies $\mathcal{P}(\alpha, \mathfrak{a})$ for some $(\alpha, \mathfrak{a})$ in $[0,1]^{\frac{m(m+1)}{2}} \times \mathbb{R}_{+}$satisfying, for $1 \leq$ $j \leq i$

$$
\begin{gathered}
\frac{m-i-1}{m-i}<\alpha_{i j} \leq 1 \quad \text { for } \quad i=1 \ldots, m-1 \\
0 \leq \alpha_{m j} \leq 1
\end{gathered}
$$

Then, there exist real numbers $k_{1}, \ldots, k_{m}$, such that, for all $\epsilon>0$ we can find positive real numbers $\lambda, \beta, \gamma$, and 


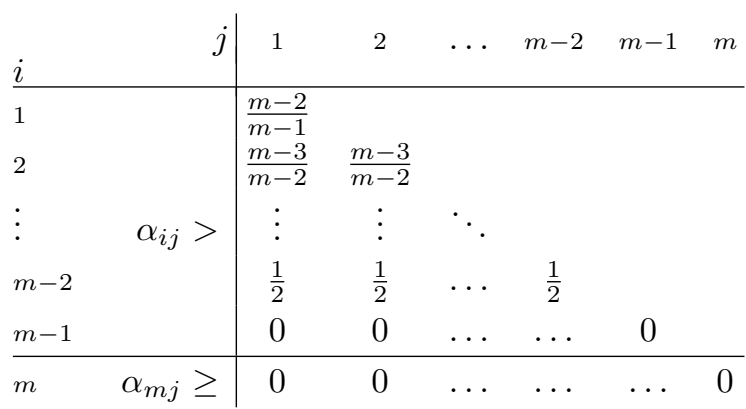

Table 1 : Hölder restrictions on $\Phi$ for arbitrarily small errors with a high gain observer.

$L^{*}$ such that, for all $L \geq L^{*}$, for all locally bounded time function $(u, v, w, \hat{w})$ and all $(z, \hat{z})$ in $\mathbb{R}^{m} \times \mathbb{R}^{m}$, any solution $\hat{Z}(\hat{z}, z, t ; u, v, w, \hat{w})$ of (4) verifies, for all $t_{0}$ and $t$ such that $t \geq t_{0} \geq 0$, and for all $i$ in $\{1, \ldots, m\}$,

$$
\begin{aligned}
& \left.\mid \hat{Z}_{i}(t)-Z_{i}(t)\right) \mid \\
& \leq \max \left\{\epsilon, L^{i-1} \beta \mid \hat{Z}_{i}\left(t_{0}\right)-Z_{i}\left(t_{0}\right)\right) \mid e^{-\lambda L\left(t-t_{0}\right)}, \\
& \left.\gamma \sup _{\substack{1 \leq j \leq m \\
s \in\left[t_{0}, t\right]}}\left\{L^{i-1}|v(s)|, \frac{\left|\Delta w_{j}(s)\right|}{L^{j-i+1}}\right\}\right\}
\end{aligned}
$$

where we have used the abbreviation $Z(t)=Z(z, t ; u, w)$ and $\hat{Z}(t)=\hat{Z}(z, \hat{z}, t ; u, v, w, \hat{w})$.

Comparing this inequality with (6), we have now the arbitrarily small non zero $\varepsilon$ in the right hand side but this is obtained under the Hölder condition instead of the Lipschitz one.

PROOF. With Young's inequality, we obtain from (3) that, for all $\sigma_{i j}$ in $\mathbb{R}_{+}$and all $\hat{\mathbf{z}}$ and $\mathbf{z}$ in $\mathbb{R}^{m}$

$$
\left|\Phi_{i}\left(u, \hat{\mathbf{z}}_{i}\right)-\Phi_{i}\left(u, \mathbf{z}_{i}\right)\right| \leq \sum_{j=1}^{i} \mathfrak{a}_{i j}\left|\hat{z}_{j}-z_{j}\right|+\mathfrak{b}_{i j},
$$

with $\mathfrak{a}_{i j}$ and $\mathfrak{b}_{i j}$ defined as

$$
\begin{cases}\mathfrak{a}_{i j}=0, \mathfrak{b}_{i j}=\mathfrak{a}, & \text { if } \alpha_{i j}=0 \\ \mathfrak{a}_{i j}=\mathfrak{a}^{\frac{1}{\alpha_{i j}}} \alpha_{i j} \sigma_{i j}^{\frac{1}{\alpha_{i j}}}, \mathfrak{b}_{i j}=\frac{1-\alpha_{i j}}{\frac{1}{\sigma_{i j}^{1-\alpha_{i j}}}} & \text { if } 0<\alpha_{i j}<1 \\ \mathfrak{a}_{i j}=\mathfrak{a}, \mathfrak{b}_{i j}=0 & \text { if } \alpha_{i j}=1\end{cases}
$$

With (8), the assumptions of Proposition 1 are satisfied with $\mathfrak{b}_{i}=\sum_{j=1}^{i} \mathfrak{b}_{i j}$. It gives $k_{1}, \ldots, k_{m}, L^{*}, \lambda, \beta$ and $\gamma$ and, if $L>\max _{i \geq j}\left\{\mathfrak{a}_{i j} L^{*}, 1\right\}$, the solution satisfies the ISS inequality (6). The result will follow if there exist $L$ and $\sigma_{i j}$ such that

$$
L>\max _{i \geq j}\left\{\mathfrak{a}_{i j} L^{*}, 1\right\}, \quad \max _{i, j} \sum_{\ell=1}^{j} \gamma \mathfrak{b}_{j \ell} L^{i-j-1} \leq \epsilon .
$$

At this point, we have to work with the expressions of $\mathfrak{a}_{i j}$ and $\mathfrak{b}_{j \ell}$ given in (9). From (7), $\alpha_{i j}$ can be zero only if $i=m$. And, when $\alpha_{m \ell}=0$, we get

$$
\gamma \mathfrak{b}_{m \ell} L^{i-m-1}=\gamma \mathfrak{a} L^{i-m-1} \leq \frac{\gamma \mathfrak{a}}{L}
$$

Say that we pick $\sigma_{m \ell}=1$ in this case. For all the other cases, we choose

$$
\sigma_{j \ell}=\left(\frac{2 j \gamma}{\epsilon}\left(1-\alpha_{j \ell}\right) L^{(m-j-1)}\right)^{1-\alpha_{j \ell}},
$$

to obtain from $(9)$

$$
\gamma \mathfrak{b}_{j \ell} L^{i-j-1} \leq \epsilon \frac{1}{j} \frac{1}{2 L^{m-i}}
$$

So, with this selection of the $\sigma_{j \ell}$, the right inequality in (10) is satisfied for $L$ sufficiently large. Then, according to $(9)$, the $a_{i j}$ are independent of $L$ or proportional to $L^{(m-i-1) \frac{1-\alpha_{i j}}{\alpha_{i j}}}$. But with $(7)$ we have

$$
0<(m-i-1) \frac{1-\alpha_{i j}}{\alpha_{i j}}<1 .
$$

This implies that $\frac{\mathfrak{a}_{i j}}{L}$ tends to 0 as $L$ tends to $+\infty$. We conclude that (10) holds if we pick $L$ sufficiently large.

It is interesting to remark the weakness of the assumptions imposed on the last two components of the function $\Phi$. Indeed, (7) only imposes that $\Phi_{m-1}$ be Hölder without any restriction on the order, and that $\Phi_{m}$ be bounded ${ }^{\text {; }}$.

\section{Cascaded high gain observer}

According to Proposition 2, the classical high gain observer can provide an arbitrary small error when the last nonlinearity is only bounded and when there is no disturbance. We exploit here this observation by proposing the following cascaded high gain observer to deal with the case where the functions $\Phi_{i}$ do not satisfy (7):

$$
\begin{aligned}
& \dot{\hat{z}}_{11}=\hat{w}_{1}-L_{1} k_{11}\left(\hat{z}_{11}-z_{1}\right) \\
& \dot{\hat{z}}_{21}=\hat{z}_{22}+\Phi_{1}\left(u, \hat{z}_{11}\right)+\hat{w}_{1}-L_{2} k_{21}\left(\hat{z}_{21}-z_{1}\right) \\
& \dot{\hat{z}}_{22}=\hat{w}_{2}-L_{2}^{2} k_{22}\left(\hat{z}_{21}-z_{1}\right) \\
& \cdots \\
& \begin{array}{c}
\dot{\hat{z}}_{m 1}=\hat{z}_{m 2}+\Phi_{1}\left(u, \hat{z}_{(m-1) 1}\right)+\hat{w}_{1}-L_{m} k_{m 1}\left(\hat{z}_{m 1}-z_{1}\right) \\
\dot{\hat{z}}_{m 2}=\hat{z}_{m 3}+\Phi_{2}\left(u, \hat{z}_{(m-1) 1}, \hat{z}_{(m-1) 2}\right) \\
\quad+\hat{w}_{2}-L_{m}^{2} k_{m 2}\left(\hat{z}_{m 1}-z_{1}\right) \\
\vdots \\
\dot{\hat{z}}_{m m}=\hat{w}_{m}-L_{m}^{m} k_{m m}\left(\hat{z}_{m 1}-z_{1}\right)
\end{array}
\end{aligned}
$$

with the gain $k_{i j}$ chosen as in a classical high gain ob- 
server of dimension $i, \hat{w}_{i}$ are estimations of $w_{i}$ and $L_{i}$ are the high gains parameters to be chosen.

Assuming the input function and the system solution are bounded, it is shown in the following that estimation with an arbitrary small error can be achieved by the cascaded high-gain observer.

Proposition 3 Assume $\Phi$ is continuous. For any positive real numbers $\bar{z}$ and $\bar{u}$, for any strictly positive real number $\epsilon$, there exist a choice of $\left(L_{1}, \ldots, L_{m}\right)$, a class $\mathcal{K} \mathcal{L}$ function $\beta$ and two class $\mathcal{K}_{\infty}$ functions $\gamma_{1}$ and $\gamma_{2}$ such that, for all locally bounded time function $(u, v, w, \hat{w})$, for all $(z, \hat{z})$ in $\mathbb{R}^{m} \times \mathbb{R}^{m}$ and for all $t$ such that $|Z(z, s ; u, w)| \leq \bar{z}$ and $|u(s)| \leq \bar{u}$ for all $0 \leq s \leq t$, any solution $\left(\hat{\mathbf{Z}}_{1}(\hat{z}, z, t ; u, v, w, \hat{w}), \ldots, \hat{\mathbf{Z}}_{m}(\hat{z}, z, t ; u, v, w, \hat{w})\right)$ of (11) verifies, for all $i$ in $\{1, \ldots, m\}$,

$$
\begin{aligned}
& \left|\hat{\mathbf{Z}}_{i}(t)-\mathbf{Z}_{i}(t)\right| \\
& \quad \leq \max \left\{\varepsilon, \beta\left(\sum_{j=1}^{i}\left|\hat{z}_{j}-z_{j}\right|, t\right),\right. \\
& \left.\sup _{s \in[0, t]}\left\{\gamma_{1}(|v(s)|), \gamma_{2}(|\Delta w(s)|)\right\}\right\}
\end{aligned}
$$

where $\hat{\mathbf{Z}}_{i}$ is the state of the ith block (see Notation 2) and we have used the abbreviation $\hat{\mathbf{Z}}_{i}(t)=$ $\hat{\mathbf{Z}}_{i}(\hat{z}, z, t ; u, v, w, \hat{w})$ and $\mathbf{Z}_{i}(t)=\mathbf{Z}_{i}(z, t ; u, w)$.

PROOF. This result is nothing but a straightforward consequence of the fact that a cascade of ISS systems is ISS.

Specifically the error system attached to the high gain observer in block $i$ has state $\mathbf{e}_{i}$ (see Notation 2) and input $v$ and $\delta_{i j}$ defined as

$$
\begin{aligned}
\delta_{i j} & =\left[\Phi_{j}\left(u, \hat{\mathbf{z}}_{(i-1)}\right)-\Phi_{j}\left(u, \mathbf{z}_{(i-1)}\right)\right]+\left[\hat{w}_{j}-w_{j}\right] \\
\delta_{i i} & =-z_{i+1}-\Phi_{i}\left(u, \mathbf{z}_{i}\right)+\hat{w}_{i}-w_{i}
\end{aligned}
$$

with $z_{m+1}=0$. With Proposition 1 , we have the existence of $k_{i 1}, \ldots, k_{i i}, \lambda_{i}, \beta_{i}$ and $\gamma_{i}$ such that we have, for all $L_{i} \geq 1$, all $t \geq t_{i} \geq 0$, all $j$ in $\{1, \ldots, i\}$ and with $e_{i j}(t)$ denoting the $j$ th error in the $i$ th block evaluated along the solution at time $t$,

$$
\begin{aligned}
& \left|e_{i j}(t)\right| \\
& \leq \max \left\{L_{i}^{j-1} \beta_{i}\left|\mathbf{e}_{i}\left(t_{i}\right)\right| e^{-\lambda_{i} L_{i}\left(t-t_{i}\right)},\right. \\
& \left.\gamma_{i} \sup _{\substack{1 \leq \ell \leq j \\
s \in\left[t_{i}, t\right]}}\left\{L_{i}^{j-1}|v(s)|, \frac{\left|\delta_{i \ell}(s)\right|}{L_{i}^{\ell-j+1}}\right\}\right\} .
\end{aligned}
$$

But the continuity of the $\Phi_{j}$ implies the existence of a function ${ }^{2} \rho$ of class $\mathcal{K}$ such that, for all $j$ in $\{1, \ldots, m\}$

\footnotetext{
${ }^{2}$ Simply take $\rho(s)=\max _{|u| \leq \overline{\mathbf{u}},\left|\mathbf{z}_{j}\right| \leq \overline{\mathbf{z}},|e| \leq s} \mid \Phi_{j}\left(u, \mathbf{z}_{j}+e\right)-$ $\Phi_{j}\left(u, \mathbf{z}_{j}\right) \mid$.
}

and for all $\left(\mathbf{z}_{(i-1)}, \hat{\mathbf{z}}_{(i-1)}, u\right)$ in $\mathbb{R}^{i-1} \times \mathbb{R}^{i-1} \times U$ satisfying $\left|\mathbf{z}_{(i-1)}\right| \leq \overline{\mathrm{z}}$ and $|u| \leq \overline{\mathrm{u}}$,

$$
\left|\Phi_{j}\left(u, \hat{\mathbf{z}}_{(i-1)}\right)-\Phi_{j}\left(u, \mathbf{z}_{(i-1)}\right)\right| \leq \rho\left(\left|\mathbf{e}_{(i-1)}\right|\right) .
$$

This implies

$\left|\delta_{i \ell}(s)\right| \leq \rho\left(\left|\mathbf{e}_{i-1}(s)\right|\right)+\left|\Delta w_{\ell}(s)\right|, \ell=1, \ldots, j-1$,

$\left|\delta_{i i}(s)\right| \leq \overline{\mathrm{z}}_{i+1}+\bar{\Phi}_{i}+\left|\Delta w_{i}(s)\right|$,

where $\bar{\Phi}_{i}=\max _{|u| \leq \overline{\mathbf{u}},\left|\mathbf{z}_{i}\right| \leq \overline{\mathbf{z}}}\left|\Phi_{i}\left(u, \mathbf{z}_{i}\right)\right|$. Hence, we have the existence of $c_{i}$ independent of $L_{i}$ such that

$$
\begin{aligned}
& \left|\mathbf{e}_{i}(t)\right| \\
& \leq c_{i} \max \left\{L_{i}^{i-1}\left|\mathbf{e}_{i}\left(t_{i}\right)\right| e^{-\lambda_{i} L_{i}\left(t-t_{i}\right)}, \sup _{s \in\left[t_{i}, t\right]} L_{i}^{i-1}|v(s)|,\right. \\
& \left.\sup _{s \in\left[t_{i}, t\right]} \frac{\rho\left(\left|\mathbf{e}_{i-1}(s)\right|\right)}{L_{i}^{2-i}}, \sup _{\substack{1 \leq \ell \leq i \\
s \in\left[t_{i}, t\right]}} \frac{\left|\Delta w_{\ell}(s)\right|}{L_{i}^{\ell-i+1}}, \frac{\overline{\mathbf{z}}_{i+1}+\bar{\Phi}_{i}}{L_{i}}\right\} .
\end{aligned}
$$

This makes precise what we wrote above that we have a cascade of ISS systems. Hence (see [23, Prop. 7.2]), for each $i$ in $\{1, \ldots, m\}$, there exist a class $\mathcal{K} \mathcal{L}$ function $\bar{\beta}_{i}$ and class $\mathcal{K}$ functions $\gamma_{v i}$ and $\gamma_{w i}$, each depending on $L_{1}$ to $L_{i}$ and such that we have, for all $t \geq 0$,

$$
\begin{aligned}
\left|\mathbf{e}_{i}(t)\right| \leq \max & \left\{\bar{\beta}_{i}\left(\max _{j \in\{1, \ldots, i\}}\left\{\left|\mathbf{e}_{j}(0)\right|\right\}, t\right),\right. \\
& \left.\varpi_{i}, \sup _{s \in[0, t]}\left\{\gamma_{v i}(|v(s)|), \gamma_{w i}(|\Delta w(s)|)\right\}\right\} .
\end{aligned}
$$

where $\varpi_{i}$ is a positive real number defined by the sequences

$$
\varpi_{1}=c_{1} \frac{\overline{\mathrm{z}}_{2}+\bar{\Phi}_{1}}{L_{1}}, \varpi_{i}=c_{i} \max \left\{\frac{\overline{\mathrm{z}}_{i+1}+\bar{\Phi}_{i}}{L_{i}}, \frac{\rho\left(\varpi_{i-1}\right)}{L_{i}^{2-i}}\right\} .
$$

Then by picking $L_{i} \geq L_{i}^{*}$ where $L_{i}^{*}$ is defined recursively as :

$$
\begin{gathered}
\epsilon_{m}=\epsilon, \quad \epsilon_{i}=\min \left(\epsilon, \rho^{-1}\left(\frac{\epsilon_{i+1}}{c_{i+1} L_{i+1}^{i-2}} \epsilon_{i+1}\right)\right) \\
L_{m}^{*}=\frac{{ }_{m} \bar{\Phi}_{m}}{\varepsilon_{m}}, \quad L_{i}^{*}=\frac{c_{i}\left[\overline{\mathbf{z}}_{i+1}+\bar{\Phi}_{i}\right]}{\varepsilon_{i}}
\end{gathered}
$$

we obtain $\varpi_{i} \leq \epsilon$ for all $i$, hence the result.

This observer has the advantage of working without any assumption on the nonlinearities besides their continuity. Note however that it requires the knowledge of a bound on the system solution and on the input. Also we may not need to build $m$ blocks, since according to Proposition 2, we need to create a new block only for the indexes $i$ where $\Phi_{i}$ does not verify Property $\mathcal{P}(\alpha, \mathfrak{a})$ for any $\mathfrak{a} \geq 0$ and with $\alpha$ satisfying (7). Unfortunately, as it appears from the proof of Proposition 3, the choice of 
$\left(L_{1}, \ldots, L_{m}\right)$ can be complicated. Besides, only a convergence with an arbitrary small error is obtained. It may thus be necessary to take very high gains which is problematic in terms of peaking and most importantly in presence of noise (see Section 8). In the following two sections, we move our attention to homogeneous observers, and show that they enable to obtain convergence.

\section{Homogeneous observer}

Homogeneous observers are extensions of high gain observers able to cope with some non Lipschitz functions. As mentioned in the introduction, they already have an old history (see [14, 15], 27, [16], 20], 21], [1, 2], 3] ). In our context they take the form :

$$
\begin{aligned}
\dot{\hat{z}}_{1} & =\hat{z}_{2}+\Phi_{1}\left(u, \hat{z}_{1}, t\right)+\hat{w}_{1}-L k_{1}\left\lfloor\hat{z}_{1}-y\right\rceil^{\frac{r_{2}}{r_{1}}} \\
\dot{\hat{z}}_{2} & =\hat{z}_{3}+\Phi_{2}\left(u, \hat{z}_{1}, \hat{z}_{2}, t\right)+\hat{w}_{2}-L^{2} k_{2}\left\lfloor\hat{z}_{1}-y\right\rceil^{\frac{r_{3}}{r_{1}}} \\
& \vdots \\
\dot{\hat{z}}_{m} & =\Phi_{m}(u, \hat{z}, t)+\hat{w}_{m}-L^{m} k_{m}\left\lfloor\hat{z}_{1}-y\right\rceil^{\frac{r_{m}+1}{r_{1}}}
\end{aligned}
$$

where $r$ is a vector in $\mathbb{R}^{m+1}$, called weight vector, the components of which, called weights, are defined by

$$
r_{i}=1-d_{0}(m-i)
$$

and where $L$ and the $k_{i}$ 's are gains to be tuned, $d_{0}$ is a parameter to be chosen in $[-1,0]$. We refer to Notation (1) for the case $d_{0}=-1$, for which the dynamics (12) must be understood as a differential inclusion. When $d_{0}=0$, we recover the high-gain observer studied in Section 3. As mentioned in Proposition 2, the usual high-gain observer can provide an estimation with an arbitrary small error provided the nonlinearity satisfies the property $\mathcal{P}(\alpha, \mathfrak{a})$ with the $\alpha_{i j}$ verifying (7). In the following proposition we claim that asymptotic estimation may be obtained with homogeneous correction terms and when considering nonlinearities which satisfies $\mathcal{P}(\alpha, \mathfrak{a})$ with the $\alpha_{i j}$ verifying

$$
\alpha_{i j}=\frac{1-d_{0}(m-i-1)}{1-d_{0}(m-j)}=\frac{r_{i+1}}{r_{j}}, 1 \leq j \leq i \leq m
$$

Those conditions in the extreme case where $d_{0}=-1$ are summed up in Table 2 . On top of that, finite time estimation may be obtained.

Proposition 4 Assume that there exist $d_{0}$ in $[-1,0]$ and $\mathfrak{a}$ in $\mathbb{R}_{+}$such that $\Phi$ satisfies $\mathcal{P}(\alpha, \mathfrak{a})$ with $\alpha$ verifying

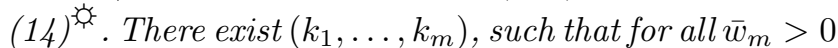
there exist $L^{*} \geq 1$ and a positive constant $\gamma$ such that, for all $L \geq L^{*}$ there exists a class $\mathcal{K} \mathcal{L}$ function $\beta$ such that for all locally bounded time function $(u, v, w, \hat{w})$, and all $(z, \hat{z})$ in $\mathbb{R}^{m} \times \mathbb{R}^{m}$ system (12) admits absolutely continuous solutions $\hat{Z}(\hat{z}, z, t ; u, v, w, \hat{w})$ defined on $\mathbb{R}_{+}$and for any such solution the following implications hold for all $t_{0}$ and $t$ such that $t \geq t_{0} \geq 0$, and for all $i$ in $\{1, \ldots, m\}$ : If $d_{0}>-1$ :

\begin{tabular}{lc|cccccc}
$i$ & & 1 & 2 & $\ldots$ & $m-2$ & $m-1$ & $m$ \\
\hline 1 & & $\alpha_{i j}=$ & $\frac{m-1}{m}$ & & & & \\
2 & & $\frac{m-2}{m}$ & $\frac{m-2}{m-1}$ & & & & \\
$\vdots$ & $\vdots$ & $\ddots$ & & & \\
$m-2$ & & $\frac{2}{m}$ & $\frac{2}{m-1}$ & $\ldots$ & $\frac{2}{3}$ & & \\
$m-1$ & $\frac{1}{m}$ & $\frac{1}{m-1}$ & $\ldots$ & $\ldots$ & $\frac{1}{2}$ & \\
$m$ & 0 & 0 & $\ldots$ & $\ldots$ & $\ldots$ & 0
\end{tabular}

Table 2 : Hölder restrictions on $\Phi$ for a homogeneous observer with $d_{0}=-1$

$$
\begin{array}{r}
\left|\hat{Z}_{i}(t)-Z_{i}(t)\right| \leq \max \left\{\beta\left(\left|\hat{Z}\left(t_{0}\right)-Z\left(t_{0}\right)\right|, t-t_{0}\right), \quad(15)\right. \\
\left.\gamma \sup _{\substack{1 \leq j \leq i \\
s \in\left[t_{0}, t\right]}}\left\{L^{i-1}|v(s)|^{\frac{r_{i}}{r_{1}}}, \frac{\left|\Delta w_{j}(s)\right|^{\frac{r_{i}}{r_{j+1}}}}{L^{\mu_{i j}}}\right\}\right\}
\end{array}
$$

where $\mu_{i j}=(j-i+1) \frac{r_{1}}{r_{j+1}}$, and we have used the abbreviation $Z(t)=Z(z, t ; u, w)$ and $\hat{Z}(t)=\hat{Z}(z, \hat{z}, t ; u, v, w, \hat{w})$. Moreover, when $d_{0}<0$ and $v(t)=w_{j}(t)=0$ for all $t$ and $j=1, \ldots, m$, there exists $T$ such that $\hat{Z}(\hat{z}, z, t)=Z(z, t)$ for all $t \geq T$.

$$
\text { If } d_{0}=-1 \text { and }\left|\Delta w_{m}(t)\right| \leq \bar{w}_{m} \text { : }
$$

$$
\begin{array}{r}
\left|\hat{Z}_{i}(t)-Z_{i}(t)\right| \leq \max \left\{\beta\left(\left|\hat{Z}\left(t_{0}\right)-Z\left(t_{0}\right)\right|, t-t_{0}\right), \quad(16)\right. \\
\left.\gamma \sup _{\substack{1 \leq j \leq i-1 \\
s \in\left[t_{0}, t\right]}}\left\{L^{i-1}|v(s)|^{\frac{r_{i}}{r_{1}}}, \frac{\left|\Delta w_{j}(s)\right|^{\frac{r_{i}}{r_{j+1}}}}{L^{\mu_{i j}}}\right\}\right\}
\end{array}
$$

where $\mu_{i j}, Z(t)$ and $\hat{Z}(t)$ are defined above.

Moreover, when $v(t)=w_{j}(t)=0$ for all $t$ and $j=$ $1, \ldots, m$, there exists $T$ such that $\hat{Z}(t)=Z(t)$ for all $t \geq T$.

Note that $j$ is in $\{1, \ldots, i\}$ in (15) whereas it is in $\{1, \ldots, i-1\}$ in $(16)$.

The proof of Proposition 4 for the case $\left.\left.d_{0} \in\right]-1,0\right]$ and without disturbances is given for example in [2. Actually [2] gives a Lyapunov design of the observer (12) with a recursive construction of both Lyapunov function and observer. Here we are concerned with the case $d_{0}=-1$. In this limit case, the observer (12) is a differential inclusion corresponding to the exact differentiator studied in [14, where convergence is established in the particular case in which $\Phi_{i}=0$ for $j=1, \ldots, m-1$ and $\Phi_{m}$ is bounded. We prove in Proposition 6 that the Lyapunov design of 2 can be extended to this case. This allows us to show that the observer (12) still converges if, for each $i, \Phi_{i}$ is Hölder with order $\alpha_{i j}$ equal to the values given in Table 2, where $i$ is the index of $\Phi_{i}$ and $j$ is the index of $e_{j}$. We also recover the same bound in presence of a noise $v$ as the one given in [14]. 
Actually some effort has been devoted to Lyapunov analysis for establishing the convergence of the observer proposed in 14. But, as far as we are aware of, this more difficult route has been successful for $m \leq 3$ only. See [19].

Finally, it is interesting to remark that in the case $d_{0}=$ -1 the ISS property between the disturbance $w_{m}$ and the estimation error is with restrictions as defined in 25, Definition 3.1]. If $\left|\Delta w_{m}(t)\right| \leq \bar{w}_{m}$ and $L$ is chosen sufficiently large, then asymptotic convergence is obtained. However, nothing can be said when $\left|\Delta w_{m}\right|>\bar{w}_{m}$. Moreover, it may be possible for a bounded large disturbance to induce a norm of the estimation error which goes to infinity. We believe that this problem could be solved employing homogeneous in the bi-limit observer as in [2]. It is shown to be doable in dimension 2 in [8].

PROOF. The set-valued function $\left.e_{1} \mapsto \mid e_{1}\right\rceil^{0}$ defined in Notations 1 is upper semi-continuous and has convex and compact values. Thus, according to 9, there exist absolutely continuous solutions to (12).

Let $\mathcal{L}=\operatorname{diag}\left(1, L, \ldots, L^{m-1}\right)$. The error $e=\hat{z}-z$ produced by the observer (12) satisfies

$$
\dot{e} \in L S_{m} e+\delta+L \mathcal{L} \mathfrak{K}\left(e_{1}+v\right)
$$

where $S_{m}$ is the shifting matrix of order $m$,

$$
\delta=\Phi(u, \hat{z})+\hat{w}-\Phi(u, z)-w,
$$

and $\mathfrak{K}$ is the homogeneous correction term the components of which are defined as

$$
\left(\mathfrak{K}\left(e_{1}\right)\right)_{i}=k_{i}\left\lfloor e_{1}\right\rceil^{\frac{r_{i+1}}{r_{1}}}
$$

where $\left(k_{1}, \ldots, k_{m}\right)$ are positive real number and $r_{i}$ is defined in (13).

Let also $V: \mathbb{R}^{m} \rightarrow \mathbb{R}_{+}$be the function defined as

$$
\begin{array}{r}
V(\bar{e})=\sum_{i=1}^{m-1} \int_{\left\lfloor\bar{e}_{i+1}\right\rceil^{\frac{r_{i}}{r_{i+1}}}\left[\lfloor x\rceil^{\frac{d_{V}-r_{i}}{r_{i}}}-\left\lfloor\bar{e}_{i+1}\right\rceil^{\frac{d_{V}-r_{i}}{r_{i+1}}}\right] d x} \\
+\frac{\left|\bar{e}_{m}\right|^{d_{V}}}{d_{V}},
\end{array}
$$

where $d_{V}$ and $\ell_{i}$ are positive real numbers such that $d_{V}>$ $2 m-1$. Note that $V$ is a homogeneous function with weight vector $r$. It is nothing but the one proposed in 2 , Theorem 3.1] for designing an observer homogeneous in the bi-limit with $d_{0}$ in $\left.]-1,0\right]$. There it is shown that, by appropriately selecting the parameters $\ell_{i}$ and $k_{i}, V$ is a strict $C^{1}$ Lyapunov function homogeneous of degree $d_{V}$ for the $L$-independent auxiliary system with state $\bar{e}$ :

$$
\dot{\bar{e}} \in S_{m} \bar{e}+\mathfrak{K}\left(\bar{e}_{1}\right) .
$$

With this result in hand a robustness analysis can be carried out on a system of the form (17). In fact, the same approach can be followed for the case $d_{0}=-1$ and the following technical result is proved in Appendix B.

Lemma 1 For all $d_{0}$ in $[-1,0]$, the function $V$ defined in (18) is positive definite and there exist positive real numbers $k_{1}, \ldots k_{m}, \ell_{1}, \ldots \ell_{m}, \lambda, c_{\delta}$ and $c_{v}$ such that for all $\bar{e}$ in $\mathbb{R}^{m}, \bar{\delta}$ in $\mathbb{R}^{m}$ and $\bar{v}$ in $\mathbb{R}$ the following implication holds :

$$
\begin{aligned}
& \text { if }\left|\bar{\delta}_{i}\right| \leq c_{\delta} V(\bar{e})^{\frac{r_{i+1}}{d_{V}}}, \quad \forall i, \text { and }|\bar{v}| \leq c_{v} V(\bar{e})^{\frac{r_{1}}{d_{V}}} \text { then }{ }^{3} \\
& \max \left\{\frac{\partial V}{\partial \bar{e}}(\bar{e})\left(S_{m}(\bar{e})+\bar{\delta}+\mathfrak{K}\left(\bar{e}_{1}+\bar{v}\right)\right)\right\} \leq-\lambda V(\bar{e})^{\frac{d_{V}+d_{0}}{d_{V}}}
\end{aligned}
$$

This Lemma says $V$ is a ISS Lyapunov function for the auxiliary system (19). See 24, Proof of Lemma 2.14] for instance. Consider now the scaled error coordinates $\varepsilon=\mathcal{L}^{-1}(\hat{z}-z)$. Straightforward computations from (17) give the error system

$$
\frac{1}{L} \dot{\varepsilon} \in S_{m} \varepsilon+\mathcal{D}_{L}+\mathfrak{K}\left(\varepsilon_{1}+v\right)
$$

with $\mathcal{D}_{L}=\mathcal{L}^{-1} \delta$. Since $\Phi$ satisfies $\mathcal{P}(\alpha, \mathfrak{a})$, with (14) and $\frac{r_{i+1}}{r_{j}} \leq 1$, we obtain, for all $L \geq 1$

$$
\begin{aligned}
\left|\mathcal{D}_{L, i}\right| & \leq \frac{\mathfrak{a}}{L} \sum_{j=1}^{i} L^{(j-1) \frac{r_{i+1}}{r_{j}}-i+1}\left|\varepsilon_{j}\right|^{\frac{r_{i+1}}{r_{j}}}+\frac{\left|\Delta w_{i}\right|}{L^{i}} \\
& \leq \frac{\mathfrak{a}}{L} \sum_{j=1}^{i}\left|\varepsilon_{j}\right|^{\frac{r_{i+1}}{r_{j}}}+\frac{\left|\Delta w_{i}\right|}{L^{i}} \\
& \leq \frac{c}{L} V(\varepsilon)^{\frac{r_{i+1}}{d_{V}}}+\frac{\left|\Delta w_{i}\right|}{L^{i}}
\end{aligned}
$$

where $c$ is a positive real number obtained from Lemma 3 in Appendix D. With Lemma 1, where $\bar{\delta}_{i}$ plays the role of $\mathcal{D}_{L, i}, \bar{v}$ the role of $v$ and $\bar{e}$ the role of $\varepsilon$, we obtain that, by picking $L^{*}$ sufficiently large such that $\frac{c}{L^{*}} \leq \frac{c_{\delta}}{2}$, we have, for all $L>L^{*}$,

$$
\begin{aligned}
& \text { if }\left\{\begin{aligned}
\frac{\left|\Delta w_{i}\right|}{L^{i}} & \leq \frac{c_{\delta}}{4} V(\varepsilon)^{\frac{r_{i+1}}{d_{V}}}, \forall i \\
|v| & \leq c_{v} V(\varepsilon)^{\frac{r_{1}}{d_{V}}}
\end{aligned}\right. \\
& \Rightarrow \frac{1}{L} \max \left\{\frac{\partial V}{\partial e}(\varepsilon) \dot{\varepsilon}\right\} \leq-\lambda V(\varepsilon)^{\frac{d_{V}+d_{0}}{d_{V}}} .
\end{aligned}
$$

Now, when evaluated along a solution, $\varepsilon$ gives rise to an absolutely continuous function $t \mapsto \varepsilon(t)$. Similarly the function defined by $t \mapsto \nu(t)=V(\varepsilon(t))$ is absolutely continuous. It follows that its time derivative is defined for almost all $t$ and, according to [22, p174], (20) implies, for almost all $t$,

3 Here the max is with respect to $s$ in $\left.\left\lfloor\bar{e}_{1}+\bar{v}\right)\right\rceil^{0}$ appearing in the $m$ th component $\mathfrak{K}\left(\bar{e}_{1}+\bar{v}\right)_{m}$ of $\mathfrak{K}\left(\bar{e}_{1}+\bar{v}\right)$. 
if $\left\{\begin{aligned} \frac{\left|\Delta w_{i}\right|}{L^{i}} & \leq \frac{c_{\delta}}{4} \nu(t)^{\frac{r_{i+1}}{d_{V}}}, \forall i \\ |v| & \leq c_{v} \nu(t)^{\frac{r_{1}}{d_{V}}}\end{aligned}\right.$

$$
\Rightarrow \frac{1}{L} \dot{\nu}(t) \leq-\lambda \nu(t)^{\frac{d_{V}+d_{0}}{d_{V}}} .
$$

Here two cases have to be distinguished.

(1) If $d_{0}$ is in ] - 1,0], with Lemma 5 in Appendix D (see also [24]), we get the existence of a class $\mathcal{K} \mathcal{L}$ function $\beta_{V}$ such that ${ }^{4}$

$$
\begin{aligned}
V(\varepsilon(t)) \leq & \max _{i \in[1, m]}\left\{\beta_{V}(V(\varepsilon(0)), \lambda L t),\right. \\
& \left.\sup _{s \in[0, t]}\left\{\left(\frac{4\left|\Delta w_{i}(s)\right|}{L^{i} c_{\delta}}\right)^{\frac{d_{V}}{r_{i+1}}}, \frac{|v(s)|^{\frac{d_{V}}{r_{1}}}}{c_{v}}\right\}\right\} .
\end{aligned}
$$

The result holds since with Lemma 3 there exist a positive real number $c_{1}$ such that

$$
\left|\frac{e_{i}}{L^{i-1}}\right| \leq c_{1} V(\epsilon)^{\frac{r_{i}}{d_{V}}} .
$$

Moreover, when $v(t)=\Delta w_{j}(t)=\mathfrak{b}_{j}=0$ for $j=1, \ldots, m,(21)$ implies finite time convergence in the case in which $d_{0}<0$.

(2) If $d_{0}=-1$, then $r_{m+1}=0$. We choose $L^{*}$ sufficiently large to satisfy

$$
\frac{\bar{w}_{m}}{\left(L^{*}\right)^{m}} \leq \frac{c_{\delta}}{4}
$$

We obtain that the first condition in (21) is satisfied for $i=m$ when $L \geq L^{*}$. With Lemma 5 in Appendix D (see also 24), the implication (21) implies the existence of a class $\mathcal{K} \mathcal{L}$ function $\beta_{V}$ such that ${ }^{4}$

$$
\begin{aligned}
V(\varepsilon(t)) \leq & \max _{i \in[1, m-1]}\left\{\beta_{V}(V(\varepsilon(0)), \lambda L t),\right. \\
& \left.\sup _{s \in[0, t]}\left\{\left(\frac{4\left|\Delta w_{i}(s)\right|}{L^{i} c_{\delta}}\right)^{\frac{d_{V}}{r_{i+1}}}, \frac{|v(s)|^{\frac{d_{V}}{r_{1}}}}{c_{v}}\right\}\right\} .
\end{aligned}
$$

And the result holds as in the previous case.

\section{Cascade of homogeneous observers}

When we cannot find $d_{0}$ in $[-1,0]$ and $\mathfrak{a}$ such that the nonlinearities satisfy $\mathcal{P}(\alpha, \mathfrak{a})$, with $\alpha$ defined in (14), we may lose the convergence of observer (12), or the possibility of making the final error arbitrarily small. In such a bad case, we can still take advantage of the fact that, for

\footnotetext{
4 according to Lemma $5, \beta_{V}(s, t)=\max \left\{0, s^{\frac{-d_{0}}{d_{V}}}-t\right\}^{\frac{d_{V}}{-d_{0}}}$
}

$\alpha$ verifying (14) with $d_{0}=-1, \mathcal{P}(\alpha, \mathfrak{a})$ does not impose any restriction besides boundedness of the last functions $\Phi_{m}$ (see Table 2).

From the remark that observer (12)

(1) can be used for the system

$$
\begin{aligned}
\dot{z}_{1} & =z_{2}+\psi_{1}(t) \\
& \vdots \\
\dot{z}_{k-1} & =z_{k}+\psi_{k-1}(t) \\
\dot{z}_{k} & =\varphi_{k}(t)
\end{aligned}
$$

provided the functions $\psi_{i}$ are known and the function $\varphi_{k}$ is unknown but bounded, with known bound.

(2) gives estimates of the $z_{i}$ 's in finite time,

we see that it can be used as a preliminary step to deal with the system

$$
\begin{aligned}
\dot{z}_{1} & =z_{2}+\psi_{1}(t) \\
& \vdots \\
\dot{z}_{k-1} & =z_{k}+\psi_{k-1}(t) \\
\dot{z}_{k} & =z_{k+1}+\Phi_{k}\left(u, z_{1}, \ldots, z_{k}\right) \\
\dot{z}_{k+1} & =\varphi_{k+1}\left(u, z_{1}, \ldots, z_{k+1}\right)
\end{aligned}
$$

Indeed, thanks to the above observer we know in finite time the values of $z_{1}, \ldots, z_{k}$, so that the function $\Phi_{k}\left(u, z_{1}, \ldots, z_{k}\right)$ becomes a known signal $\psi_{k}(t)$.

From this, we can propose the following observer made of a cascade of homogeneous observers :

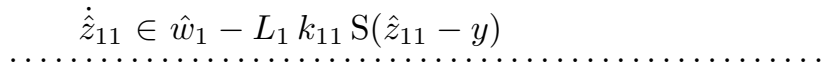

$$
\begin{aligned}
& \dot{\hat{z}}_{21}=\hat{z}_{22}+\Phi_{1}\left(u, \hat{z}_{11}\right)+\hat{w}_{1}-L_{2} k_{21}\left\lfloor\hat{z}_{21}-y\right\rceil^{\frac{1}{2}} \\
& \dot{\hat{z}}_{22} \in \hat{w}_{2}-L_{2}^{2} k_{22} \mathrm{~S}\left(\hat{z}_{21}-y\right)
\end{aligned}
$$

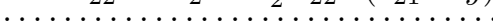

$$
\begin{aligned}
& \dot{\hat{z}}_{m 1}=\hat{z}_{m 2}+\Phi_{1}\left(u, \hat{z}_{11}\right) \\
& +\hat{w}_{1}-L_{m} k_{m 1}\left\lfloor\hat{z}_{m 1}-y\right\rceil^{\frac{m-1}{m}} \\
& \dot{\hat{z}}_{m(m-1)} \stackrel{\vdots}{=} \hat{z}_{m m}+\Phi_{m-1}\left(u, \hat{z}_{(m-1) 1}, \ldots, \hat{z}_{(m-1)(m-1)}\right) \\
& +\hat{w}_{m-1}-L_{m}^{m-1} k_{m(m-1)}\left\lfloor\hat{z}_{m 1}-y\right\rceil^{\frac{1}{m}} \\
& \dot{\hat{z}}_{m m} \in \hat{w}_{m}-L_{m}^{m} k_{m m} \mathrm{~S}\left(\hat{z}_{m 1}-y\right)
\end{aligned}
$$

where the $k_{i j}$ and $L_{i}$ are positive real numbers to be tuned.

As a direct consequence of Proposition 4 and following the same steps as in the proof of Proposition 3, we have 
Proposition 5 Assume $\Phi$ is continuous. For any positive real numbers $\bar{z}, \bar{u} \bar{w}$, we can find positive real numbers $k_{i j}$ and $L_{i}$, two class $\mathcal{K}$ functions $\gamma_{1}$ and $\gamma_{2}$ and a class $\mathcal{K} \mathcal{L}$ function $\beta$ such that, for all locally bounded time function $(u, v, w, \hat{w})$, and all $(z, \hat{z})$ in $\mathbb{R}^{m} \times \mathbb{R}^{m}$, the observer (22) admits absolutely continuous solutions $\left(\hat{\mathbf{Z}}_{1}(\hat{z}, z, t ; u, v, w, \hat{w}), \ldots, \hat{\mathbf{Z}}_{m}(\hat{z}, z, t ; u, v, w, \hat{w})\right) \quad$ which are defined on $\mathbb{R}_{+}$and for any such solution we have for all $i$ in $\{1, \ldots, m\}$ and for all t such that $|Z(z, s ; u, w)| \leq \bar{z}$, $|u(s)| \leq \bar{u}$ and $|\Delta w(s)| \leq \bar{w}$ for all $0 \leq s \leq t$ :

$$
\begin{aligned}
\left|\hat{\mathbf{Z}}_{i}(t)-\mathbf{Z}_{i}(t)\right| \leq \max \{\beta(|z-\hat{z}|, t), & \left.\sup _{\substack{1 \leq j \leq i-1 \\
s \in\left[t_{0}, t\right]}}\left\{\gamma_{1}(|v(s)|), \gamma_{2}\left(\left|\Delta w_{j}(s)\right|\right)\right\}\right\} .
\end{aligned}
$$

where $\hat{\mathbf{Z}}_{i}$ is the state of the ith block (see Notation 2) and we have used the abbreviation $\hat{\mathbf{Z}}_{i}(t)=$ $\hat{\mathbf{Z}}_{i}(\hat{z}, z, t ; u, v, w, \hat{w})$ and $\mathbf{Z}_{i}(t)=\mathbf{Z}_{i}(z, t ; u, w)$.

Moreover, when $v(t)=\Delta w_{j}=0$, there exists $T$ such that $\hat{\mathbf{Z}}_{i}(\hat{z}, z, t)=\mathbf{Z}_{i}(z, t)$ for all $t \geq T$.

This observer is an extension of the cascaded high gain observer (11) presented in Section 4. The use of homogeneity enables here to obtain convergence without demanding anything but the knowledge of a bound on the input and on the system solution. A drawback of a cascade of observers is that it gives an observer with dimension $\frac{m(m+1)}{2}$ in general. However, as seen in Section 4 , it may be possible to reduce this dimension since, for each new block, one may increase the dimension by more than one, when the corresponding added functions $\Phi_{i}$ satisfy $\mathcal{P}(\alpha, \mathfrak{a}) \mathrm{m}$ for some $\alpha$ verifying (14) with $d_{0}=-1$ and for some $\mathfrak{a}$.

Finally, note that the result of Proposition 5 does not mean that the observer is ISS with respect to $\Delta w$. Indeed, $\Delta w$ must be bounded to obtain this ISS-like inequality : the system is ISS with restrictions. Again, we believe that this problem could be solved employing homogeneous in the bi-limit observer as in [2].

\section{Relaxing the assumptions marked with}

First, if System (2) is not complete, every ISS inequalities still holds for any solution $Z(z, t ; u, w)$ but only on $[0, T(z)[$ where $T(z)$ is its maximal time of existence.

The global aspect of boundedness, Höder, $\mathcal{P}(\alpha, \mathfrak{a}), \ldots$, can be relaxed as follows. Let $U$ be bounded and let $\mathcal{M}$ be a given compact set. We define $\hat{\Phi}$, to be used instead of $\Phi$ in the observers, as

$$
\hat{\Phi}_{i}\left(u, z_{1}, \ldots, z_{i}\right)=\operatorname{sat}\left(\Phi_{i}\left(u, z_{1}, \ldots, z_{i}\right), \bar{\Phi}_{i}\right)
$$

where $\bar{\Phi}_{i}=\max _{u \in U, z \in \mathcal{M}}\left(\Phi_{i}\left(u, z_{1}, \ldots, z_{i}\right)\right)$ and the saturation function is defined on $\mathbb{R}$ by

$$
\operatorname{sat}(x, M)=\max (\min (x, M),-M) .
$$

It can be shown that, for any compact set $\tilde{\mathcal{M}}$ strictly contained in $\mathcal{M}$, there exists $\tilde{\mathfrak{a}}$ such that (3) holds for $\hat{\Phi}$ for all $\left(\mathbf{z}_{a}, \mathbf{z}_{b}\right)$ in $\mathbb{R}^{m} \times \tilde{\mathcal{M}}$. Then, since $\hat{\Phi}=\Phi$ on $\tilde{\mathcal{M}}$, we can modify the assumptions

- in Proposition 1, so that (5) holds only on the compact set $\mathcal{M}$;

- in Propositions 2 and 4, so that $\Phi$ verifies $\mathcal{P}(\alpha, \mathfrak{a})$ only on the compact set $\mathcal{M}$;

- Propositions 3 and 5 remain unchanged.

In this case, the results hold for the particular system solutions $Z(z, t ; u, w)$ which are in the compact set $\tilde{\mathcal{M}}$ for $t$ in $[0, T(z)$ [. Precisely, for these solutions, the bounds on $\hat{Z}_{i}(t)-Z_{i}(t)$ given in these Propositions hold for all $t$ in $[0, T(z)[$.

Note also that if $\mathcal{P}(\alpha, \mathfrak{a})$ holds on a compact set, then for any $\tilde{\alpha}$ such that $\tilde{\alpha}_{i j} \leq \alpha_{i j}$ for all $(i, j)$, there exists $\tilde{a}$ such that $\mathcal{P}(\tilde{\alpha}, \tilde{\mathfrak{a}})$ also holds on this compact set. It follows that the constraints given by (14) or Table 2 in Proposition 4 can be relaxed to $\alpha_{i j} \geq \frac{1-d_{0}(m-i-1)}{1-d_{0}(m-j)}$, and the less restrictive conditions one may ask for are obtained for $d_{0}=-1$.

Finally, in Propositions 1, 2 and 4, it is possible to consider the case where $\Phi$ depends also on time as long as any assumption made on $\Phi$ is satisfied uniformly with respect to time.

\section{Example}

Consider the system

$\dot{x}_{1}=x_{2}, \dot{x}_{2}=-x_{1}+x_{3}^{5} x_{1}, \dot{x}_{3}=-x_{1} x_{2}+u, y=x_{1}$

with $u$ as input. It would lead us too far from the main subject of this article to study here the solutions behavior of this system. We note however that, when $u$ is zero, they evolve in the 2-dimensional surface $\{x \in$ $\left.\mathbb{R}^{3}: 3 x_{1}^{2}+3 x_{2}^{2}+x_{3}^{6}=c^{6}\right\}$ which is diffeomorphic ${ }^{5}$ to the sphere $\mathbb{S}^{2}$. Thanks to Poincaré-Bendixon theory, we know the solutions are periodic and circling the unstable equilibria $\left(x_{1}=x_{2}=0, x_{3}= \pm c\right)$. So we hope for the existence of solutions remaining in the compact set

$$
\mathcal{C}_{r, \epsilon}=\left\{x \in \mathbb{R}^{3}: x_{1}^{2}+x_{2}^{2} \geq \epsilon, 3 x_{1}^{2}+3 x_{2}^{2}+x_{3}^{6} \leq r\right\}
$$

for instance when $u$ is a small periodic time function, except maybe for pairs of input $u$ and initial condition $\left(x_{1}, x_{2}, x_{3}\right)$ for which resonance could occur. Moreover, due to their periodic behavior, such solutions are likely to have their $x_{3}$ component recurrently crossing zero.

\footnotetext{
5 A diffeomorphism from the unit sphere to the set is $x \mapsto$ $x \rho(x)$ where $\rho$ is the unique positive solution (hint: $x_{3} \leq 1$ ) of $\rho^{6} x_{3}^{6}+3 \rho^{2}\left(1-x_{3}^{2}\right)-1=0$
} 


\subsection{Uniform and differential observability}

On $\mathcal{S}=\left\{x \in \mathbb{R}^{3}: x_{1}^{2}+x_{2}^{2} \neq 0\right\}$, and whatever $u$ is, the knowledge of the function $t \mapsto y(t)=X_{1}(x, t)$ and therefore of its three first derivatives

$\dot{y}=x_{2}$

$\ddot{y}=-x_{1}+x_{3}^{5} x_{1}$

$\dddot{y}=-x_{2}-5 x_{3}^{4} x_{1}^{2} x_{2}+x_{3}^{5} x_{2}+5 x_{3}^{4} x_{1} u$

gives us $x_{1}, x_{2}$ and $x_{3}$. Thus, System (24) is uniformly observable on $\mathcal{S}$. Besides, the function

$$
\mathbf{H}_{4}(x)=\left(\begin{array}{c}
x_{1} \\
x_{2} \\
-x_{1}+x_{3}^{5} x_{1} \\
-x_{2}-5 x_{3}^{4} x_{1}^{2} x_{2}+x_{3}^{5} x_{2}
\end{array}\right)
$$

is injective on $\mathcal{S}$ and admits the left following inverse, defined on $\left\{z \in \mathbb{R}^{4}: z_{1}^{2}+z_{2}^{2} \neq 0\right\}$, is:

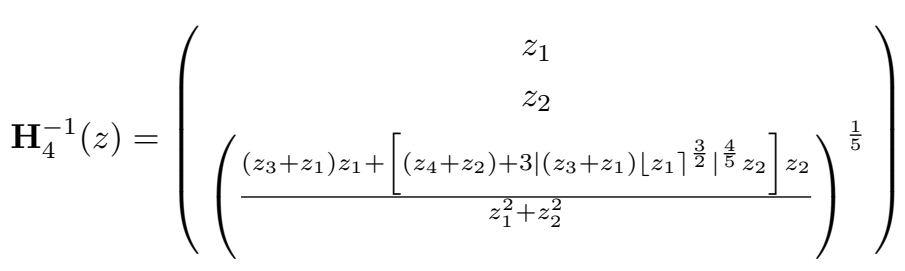

However, $\mathbf{H}_{4}$ is not an immersion because of a singularity of its Jacobian at $x_{3}=0$. So the system is differentially observable of order 4 on $\mathcal{S}$ but not strongly. According to 6], it admits a triangular canonical form of dimension 4 but with functions $\Phi$ maybe non Lipschitz.

\subsection{Triangular form and property $\mathcal{P}\left(d_{0}, c, 0\right)$}

The triangular canonical form of dimension 4 mentioned above is

$$
\begin{aligned}
\dot{z}_{1} & =z_{2} \\
\dot{z}_{2} & =z_{3} \\
\dot{z}_{3} & =z_{4}+\Phi_{3}\left(u, z_{1}, z_{2}, z_{3}\right) \\
\dot{z}_{4} & =\Phi_{4}(u, z) \\
y & =z_{1} .
\end{aligned}
$$

where $\Phi_{3}\left(u, z_{1}, z_{2}, z_{3}\right)=5 u\left|z_{3}+z_{1}\right|^{\frac{4}{5}}\left|z_{1}\right|^{\frac{1}{5}}$ and $\Phi_{4}$ is a continuous non-Lipschitz function the expressions of which is complex, fortunately with no interest here. The function $\Phi_{3}$ is not Lipschitz at the points on the hyperplanes $z_{3}=-z_{1}$ and $z_{1}=0$ (image by $\mathbf{H}_{3}$ of points where $x_{3}=0$ or $x_{1}=0$ ) known to be visited possibly recurrently along solutions. This example thus falls precisely into the scope of the paper.

The function $\Phi_{4}$ is continuous and therefore bounded on any compact set including $\mathbf{H}_{4}\left(\overline{\mathcal{C}}_{r, \epsilon}\right)$. Besides, for $\hat{\mathbf{z}}_{3}$ and $\mathbf{z}_{3}$ in a compact set including $\mathbf{H}_{3}\left(\overline{\mathcal{C}}_{r, \epsilon}\right)$, there exist

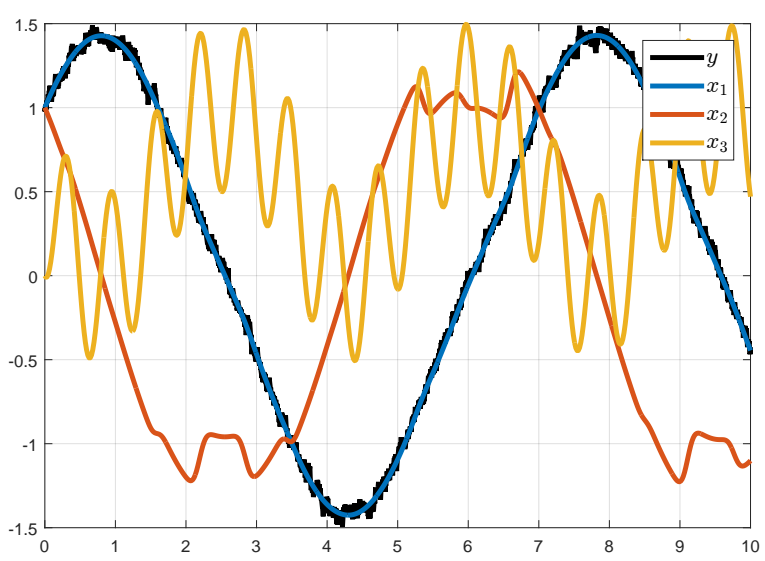

Fig. 1. Trajectory of System (24), with the noised measurement $y$.

$c_{1}$ and $c_{3}$ such that

$$
\begin{aligned}
\mid \Phi_{3}\left(u, \hat{z}_{1}, \hat{z}_{2}, \hat{z}_{3}\right)-\Phi_{3} & \left(u, z_{1}, z_{2}, z_{3}\right) \mid \\
& \leq c_{1} u\left|\hat{z}_{1}-z_{1}\right|^{\frac{1}{5}}+c_{3} u\left|\hat{z}_{3}-z_{3}\right|^{\frac{4}{5}} .
\end{aligned}
$$

This implies that $\Phi_{3}$ is Hölder with order $\frac{1}{5}$.

Hence the nonlinearities $\Phi_{3}$ and $\Phi_{4}$ verify the conditions of Table 1 . This implies that for $L$ sufficiently large, convergence with an arbitrary small error can be achieved with the high gain observer (4). However, $\Phi_{3}$ does not verify the conditions of Table 2 . Thus, there is no theoretical guarantee that the homogeneous observer (12) with $d_{0}=-1$ will provide exact convergence.

\subsection{An observer of dimension 4?}

We consider the solution to system (24) with initial condition $x=(1,1,0)$ and $u=5 \sin (10 t)$. This solution is periodic and regularly crosses the Lipschitzness singularities $x_{3}=0$ or $x_{1}=0$, as illustrated in Figure 1 . In the following, we use the same noised measurement $y$, shown on Figure 1, in every simulation with noise. It is a filtered gaussian noise with standard deviation $\sigma=0.03$ and 1st order filtering parameter $a=50$.

We first implement a high gain observer of dimension 4 , in the absence of noise, initialized at $\hat{x}=(0.1,0.1,0)$, and with the gains $k_{1}=14, k_{2}=99, k_{3}=408, k_{4}=833$. As an illustration of Proposition 2, the convergence with an arbitrary small error is achieved and is illustrated in Table 3. However, we observe that the decrease of the errors, especially for $e_{z, 4}$, is very slow compared to the increase of the peaking and a very high gain is needed to obtain "acceptable" final errors. In presence of noise, the tradeoff between final error and noise amplification becomes very difficult : with the noised measurement of Figure 1, the smallest final error $e_{z, 4}$ is 200, achieved for $L=2$. Of course, there might exist a choice of the gains $k_{i}$ giving better results. But overall a high gain observer may not be a systematic solution in practice for 
non-Lipschitz triangular systems, especially when the solution regularly crosses the Lipschitz-singularities.

\begin{tabular}{|c|c|c|c|c|c|}
\hline $\mathrm{L}$ & $e_{z, 1}$ & $e_{z, 2}$ & $e_{z, 3}$ & $e_{z, 4}$ & Peaking \\
\hline 2 & 0.15 & 4 & 60 & 200 & 300 \\
\hline 5 & $6.10^{-4}$ & 0.04 & 1.5 & 30 & 4000 \\
\hline 8 & $5.10^{-5}$ & $4.10^{-3}$ & 0.25 & 7 & $1.5 .10^{4}$ \\
\hline 10 & $8.10^{-6}$ & $1.10^{-3}$ & 0.1 & 4 & $3.5 .10^{4}$ \\
\hline 15 & $1.5 .10^{-6}$ & $3.10^{-4}$ & 0.03 & 2 & $1.2 .10^{5}$ \\
\hline
\end{tabular}

Table 3

Decrease of the final error in the $z$-coordinates $\left(e_{z, i}=\hat{z}_{i}-z_{i}\right)$ with the gain $L$, with a high gain observer and in the absence of noise.

Let us now implement an homogeneous observer of dimension 4 with an explicit Euler method with fixed measurement and integration steps equaling $10^{-5}$, and with the Matlab sign function. The degree is $d_{0}=-1$, and the gains are chosen according to [16], i-e $k_{1}=5, k_{2}=8.77$, $k_{3}=4.44, k_{4}=1.1$. For a gain $L=3$, the convergence is achieved with a final error of $8 \cdot 10^{-4}$ on $z_{4}$, even though the Hölder restriction of Proposition 4 is a priori not satisfied around $z_{1}=0$. Unfortunately, the final errors are heavily impacted in presence of noise, as illustrated in Table 4. This may also come from a lack of ISS property. Notice that the amplification of the noise by the gain $L$ is not as rapid as expected from the bound in Proposition 4. The final errors remain nonetheless too large, although, once again, we did not optimize our choice of gains $k_{i}$.

\begin{tabular}{|c|c|c|c|c|}
\hline $\mathrm{L}$ & $e_{z, 1}$ & $e_{z, 2}$ & $e_{z, 3}$ & $e_{z, 4}$ \\
\hline 2.5 & 0.15 & 3.5 & 30 & 18 \\
\hline 3 & 0.15 & 3 & 35 & 25 \\
\hline 4 & 0.1 & 2 & 25 & 50 \\
\hline 5 & 0.1 & 2 & 30 & 80 \\
\hline 6 & 0.1 & 2 & 35 & 120 \\
\hline
\end{tabular}

Table 4

Final errors in the $z$-coordinates given by a homogeneous observer of degree -1 in presence of noise.

\subsection{Cascaded observers}

In the absence of noise, the cascaded observers presented in Sections 4 and 6 give similar results to the corresponding observers in dimension 4, i-e arbitrary small asymptotic error and finite time convergence respectively. However, they seem to provide better accuracies in presence of noise.

In the case of a high gain cascade observer, the errors, although smaller than in the high gain observer of dimension 4 , remain too large to consider it a viable solution.
On the other hand, the homogeneous cascade observer :

$$
\begin{aligned}
& \dot{\hat{z}}_{11}=\hat{z}_{12}-L_{1} k_{11}\left\lfloor\hat{z}_{11}-y\right\rceil^{\frac{2}{3}} \\
& \dot{\hat{z}}_{12}=\hat{z}_{13}-L_{1}^{2} k_{12}\left\lfloor\hat{z}_{11}-y\right\rceil^{\frac{1}{3}} \\
& \dot{\hat{z}}_{13} \in-L_{1}^{3} k_{13} \mathrm{~S}\left(\hat{z}_{11}-y\right) \ldots \ldots \ldots \ldots \ldots \ldots \ldots \ldots \ldots \ldots \ldots \ldots \ldots \ldots \ldots \ldots \ldots \ldots \ldots \ldots \ldots \\
& \cdots \ldots \ldots \\
& \dot{\hat{z}}_{21}=\hat{z}_{22}-L_{2} k_{21}\left\lfloor\hat{z}_{21}-y\right\rceil^{\frac{3}{4}} \\
& \dot{\hat{z}}_{22}=\hat{z}_{23}-L_{2}^{2} k_{22}\left\lfloor\hat{z}_{21}-y\right\rceil^{\frac{1}{2}} \\
& \dot{\hat{z}}_{23}=\hat{z}_{24}+\operatorname{sat}\left(\mathfrak{g}_{3}\left(\hat{z}_{11}, \hat{z}_{12}, \hat{z}_{13}\right)\right) u-L_{2}^{3} k_{23}\left\lfloor\hat{z}_{21}-y\right\rceil^{\frac{1}{4}} \\
& \dot{\hat{z}}_{24} \in-L_{2}^{4} k_{24} \mathrm{~S}\left(\hat{z}_{21}-y\right)
\end{aligned}
$$

with the coefficients $k_{1 j}$ chosen, according to [16], as $k_{11}=3, k_{12}=2.6, k_{13}=1.1$, and $k_{2 j}$ as above, and with the gains $L_{1}=2.5$ and $L_{2}=3$, gives the following final errors :

$$
e_{z, 11}=0.05, \quad e_{z, 12}=0.4, \quad e_{z, 13}=2.5, \quad e_{z, 24}=12
$$

Comparing to Table 4, we see that implementing an intermediate homogeneous observer of dimension 3 enables to obtain much better estimates of the first three states $z_{i}$, which are then used in the nonlinearity of the second block, thus giving a better estimate of $z_{4}$.

Unfortunately, the presented results are still unsatisfactory in presence of noise, which leaves the question of the construction of robust observers for such systems unanswered.

\section{Conclusion}

To summarize the most important ideas, we provide in Table 9 a synthetic comparison of the four observers studied in this paper, in the usual case where the system state and the input are bounded.

We have shown the convergence with an arbitrary small error of the classical high gain observer in presence of nonlinearities verifying some Hölder-like condition. The same result could probably be obtained for the high gainlike observer presented in 7. Also, for the case when this Hölder condition is not verified, we proposed a novel cascaded high gain observer. Under slightly more restrictive assumptions, we proved the convergence of an homogeneous observer and of its cascaded version with the help of an explicit Lyapunov function.

Our numerical experience indicates however that to improve the performances in presence of measurement noise, it is very difficult to tune the gains of both high gain and homogeneous observers, although it is slightly simpler for the latter since smaller gains are sufficient to ensure convergence. Simulations on our example suggest that the situation may be more favorable with the cascaded homogeneous observer. Our ISS bounds in this paper being far too conservative, it is necessary to carry out a finer study if we want to optimally tune the gains of the observers. It may also be appropriate to use online gain adaptation techniques since large gains should be necessary only around the points where the nonlinearities are not Lipschitz. About these two aspects, we 
refer the reader to the survey in [13, Sections 3.2.2 and 3.2.3] and the references therein.

\section{A Barbot et al's observer}

The set valued map proposed in 4 to obtain an observer for a triangular canonical form where the functions are only locally bounded is defined as follows. Given $(\hat{z}, y, u)$, $\left(v_{1}, \ldots, v_{m}\right)$ is in $F(\hat{z}, y, u)$ if there exists $\left(\tilde{z}_{2}, \ldots, \tilde{z}_{m}\right)$ in $\mathbb{R}^{m-1}$ such that:

$$
\begin{aligned}
& v_{1}=\tilde{z}_{2}+\mathfrak{g}_{1}(y) u \\
& \tilde{z}_{2} \in \operatorname{sat}\left(\hat{z}_{2}\right)-k_{1} S\left(y-\hat{z}_{1}\right) \\
& \vdots \\
& v_{i}=\tilde{z}_{i+1}+\mathfrak{g}_{i}\left(y, \tilde{z}_{2}, \ldots, \tilde{z}_{i}\right) u \\
& \tilde{z}_{i+1} \in \operatorname{sat}\left(\hat{z}_{i+1}\right)-k_{i} S\left(\hat{z}_{i}-\tilde{z}_{i}\right) \\
& \vdots \\
& v_{m} \in \varphi_{m}\left(y, \tilde{z}_{2}, \ldots, \tilde{z}_{m}\right) \\
& \quad \quad+\mathfrak{g}_{m}\left(y, \tilde{z}_{2}, \ldots, \tilde{z}_{m}\right) u-k_{m} S\left(\hat{z}_{m}-\tilde{z}_{m}\right)
\end{aligned}
$$

where sat is some saturation function.

\section{B Proof of Lemma 1}

The proof is based on the following Proposition the proof of which is given in the following section for the case $d_{0}=-1$ and can be found for $d_{0}$ in $\left.]-1,0\right]$ in [2. This proposition establishes that for a chain of integrator it is possible to construct homogeneous correction terms which provide an observer and that it is possible to construct a smooth strict homogeneous Lyapunov function.

Proposition 6 For all $d_{0}$ in $[-1,0]$, the function $V$ defined in (18) is positive definite and there exists positive real numbers $k_{1}, \ldots k_{m}, \ell_{1}, \ldots \ell_{m}, \tilde{\lambda}$ such that for all e in $\mathbb{R}^{m}$, the following holds :

$$
\max \left\{\frac{\partial V}{\partial \bar{e}}(\bar{e})\left(S_{m}(\bar{e})+\mathfrak{K}\left(\bar{e}_{1}\right)\right)\right\} \leq-\tilde{\lambda} V(\bar{e})^{\frac{d_{V}+d_{0}}{d_{V}}} .
$$

Let $\tilde{\mathfrak{K}}\left(\bar{e}_{1}, s\right)$ be the function defined as

$$
\left(\tilde{\mathfrak{K}}\left(\bar{e}_{1}, s\right)\right)_{i}=\left(\mathfrak{K}\left(\bar{e}_{1}\right)\right)_{i}, i \in[1, m-1],
$$

and,

$$
\left(\tilde{\mathfrak{K}}\left(\bar{e}_{1}, s\right)\right)_{m}=\left\{\begin{array}{l}
k_{m} s, \text { when } d_{0}=-1 \\
\left(\mathfrak{K}\left(\bar{e}_{1}\right)\right)_{m}, \text { when } d_{0}>-1
\end{array}\right.
$$

Note that $\tilde{\mathfrak{K}}$ is a continuous (single) real-valued function which satisfies for all $\bar{e}_{1}$ in $\mathbb{R}$

$$
\mathfrak{K}\left(\bar{e}_{1}\right)=\left\{\tilde{\mathfrak{K}}\left(\bar{e}_{1}, s\right), \quad s \in \mathrm{S}\left(\bar{e}_{1}\right)\right\}
$$

Consider also the functions

$$
\begin{aligned}
\tilde{\eta}(\bar{e}, \bar{\delta}, \bar{v}, s)=\frac{\partial V}{\partial \bar{e}}(\bar{e})\left(S_{m}(\bar{e})+\bar{\delta}+\tilde{\mathfrak{K}}\left(\bar{e}_{1}+\bar{v}, s\right)\right) \\
+\frac{\tilde{\lambda}}{2} V(\bar{e})^{\frac{d_{V}+d_{0}}{d_{V}}}
\end{aligned}
$$

and

$$
\gamma(\bar{\delta}, v)=\sum_{i=1}^{m}\left|\bar{\delta}_{i}\right|^{\frac{d_{V}+d_{0}}{r_{i+1}}}+|\bar{v}|^{\frac{d_{V}+d_{0}}{r_{1}}} .
$$

With (B.1), we invoke Lemma 3 to get the existence of a positive real number $c_{1}$ satisfying :

$$
\begin{aligned}
\frac{\partial V}{\partial \bar{e}}(\bar{e})( & \left.S_{m}(\bar{e})+\Delta+\tilde{\mathfrak{K}}\left(\bar{e}_{1}+\bar{v}, s\right)\right) \\
& \leq-\frac{\tilde{\lambda}}{2} V(\bar{e})^{\frac{d_{V}+d_{0}}{d_{V}}}+c_{1} \sum_{i=1}^{m} \bar{\delta}_{i}^{\frac{d_{V}+d_{0}}{r_{i+1}}}+c_{1}|\bar{v}|^{\frac{d_{V}+d_{0}}{r_{1}}} .
\end{aligned}
$$

This can be rewritten,

$$
\begin{gathered}
\frac{\partial V}{\partial \bar{e}}(\bar{e})\left(S_{m}(\bar{e})+\bar{\delta}+\tilde{\mathfrak{K}}\left(\bar{e}_{1}+v, s\right)\right) \leq-\frac{\tilde{\lambda}}{2(m+2)} V(\bar{e})^{\frac{d_{V}+d_{0}}{d_{V}}} \\
+\sum_{i=1}^{m}\left(c_{1}\left|\bar{\delta}_{i}\right|^{\frac{d_{V}+d_{0}}{r_{i+1}}}-\frac{\tilde{\lambda}}{2(m+2)} V(\bar{e})^{\frac{d_{V}+d_{0}}{d_{V}}}\right) \\
+c_{1}|\bar{v}|^{\frac{d_{V}+d_{0}}{r_{1}}}-\frac{\tilde{\lambda}}{2(m+2)} V(\bar{e})^{\frac{d_{V}+d_{0}}{d_{V}}} .
\end{gathered}
$$

Consequently, the result holds with $\lambda=\frac{\tilde{\lambda}}{2(m+2)}, c_{\delta}=$ $c_{v}=\left(\frac{\alpha}{c_{1}}\right)^{\frac{r_{1}}{d_{V}+d_{0}}}$.

\section{Proof of Proposition 6 when $d_{0}=-1$}

In this section, we denote $E_{i}=\left(e_{i}, \ldots, e_{m}\right)$. Let $d_{V}$ be an integer such that $d_{V}>2 m-1$ and the functions $\mathfrak{K}_{i}$ recursively defined by :

$\mathfrak{K}_{m}\left(e_{m}\right)=-\left\lfloor e_{m}\right\rceil^{0} \quad, \quad \mathfrak{K}_{i}\left(e_{i}\right)=\left(\begin{array}{c}-\left\lfloor\ell_{i} e_{i}\right\rceil^{\frac{r_{i+1}}{r_{i}}} \\ \mathfrak{K}_{i+1}\left(\left\lfloor\ell_{i} e_{i}\right\rceil^{\frac{r_{i+1}}{r_{i}}}\right)\end{array}\right)$.

Let $V_{m}\left(e_{m}\right)=\frac{\left|e_{m}\right|^{d} V}{d_{V}}$ and for all $i$ in $\{1, \ldots, m-1\}$, let also $\bar{V}_{i}: \mathbb{R}^{2} \rightarrow \mathbb{R}$ and $V_{i}: \mathbb{R}^{n-i+1} \rightarrow \mathbb{R}$ be the functions defined by

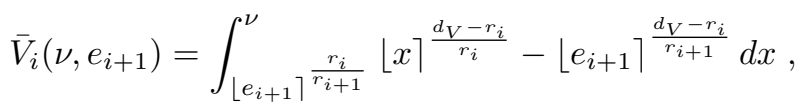

$$
\begin{aligned}
& V_{i}\left(E_{i}\right)=\sum_{j=m-1}^{i} \bar{V}_{j}\left(\ell_{j} e_{j}, e_{j+1}\right)+V_{m}\left(e_{m}\right) \text {. }
\end{aligned}
$$

With these definitions, the Lyapunov function $V$ defined in (18) is simply $V(e)=V_{1}(e)$ and the homogeneous 


\begin{tabular}{|c|c|c|c|c|}
\hline & High gain (4) & High gain cascade (11) & Homogeneous (12) & $\begin{array}{l}\text { Homogeneous cascade } \\
(22)\end{array}$ \\
\hline $\begin{array}{l}\text { Assumption } \\
\text { on } \mathfrak{g}_{i}\end{array}$ & $\begin{array}{l}\text { Hölder with order greater } \\
\text { than in Table } 1\end{array}$ & Continuous & $\begin{array}{l}\text { Hölder with order greater } \\
\text { than in (14) or Table } 2 \text { for } \\
d_{0}=-1\end{array}$ & Continuous \\
\hline Convergence & Arbitrary small error & Arbitrary small error & Asymptotic convergence & Asymptotic convergence \\
\hline Advantages & Easy choice of gains & No constraint on $\mathfrak{g}_{i}$ & $\begin{array}{l}\text { Not necessarily large } \\
\text { gains because } \\
\text { gence }\end{array}$ & $\begin{array}{l}\text { No constraint on } \mathfrak{g}_{i} \text {, con- } \\
\text { vergence, apparently bet- } \\
\text { ter in terms of noise }\end{array}$ \\
\hline Drawbacks & $\begin{array}{l}\text { Large gains necessary to } \\
\text { obtain small error } \Rightarrow \text { nu- } \\
\text { merical problems (peak- } \\
\text { ing) and sensitivity to } \\
\text { noise }\end{array}$ & $\begin{array}{l}\text { Same as for high gain, } \\
\text { but also gains difficult to } \\
\text { choose and large dimen- } \\
\text { sion }\end{array}$ & $\begin{array}{l}\text { Implementation of the } \\
\text { sign function if } d_{0}=-1 \\
\text { (chatter etc) }\end{array}$ & $\begin{array}{l}\text { Large dimension and a } \\
\text { lot of gains to choose }\end{array}$ \\
\hline
\end{tabular}

Table 5

Comparison between observers when the system state and the input are bounded.

vector field $\mathfrak{K}\left(e_{1}\right)=\mathfrak{K}_{1}\left(e_{1}\right)$ with

$$
k_{i}=\ell_{i}^{\frac{r i+1}{r_{i}}} \ell_{i-1}^{\frac{r_{i+1}}{r_{i}-1}} \ldots \ell_{2}^{\frac{r_{i+1}}{r_{2}}} \ell_{1}^{\frac{r_{i+1}}{r_{1}}} .
$$

Note that the $j$ th component of $\mathfrak{K}_{i}$ is homogeneous of degree $r_{j+1}=m-j$ and, for any $e_{i}$ in $\mathbb{R}$, the set $\mathfrak{K}_{i}\left(e_{i}\right)$ can be expressed as

$$
\mathfrak{K}_{i}\left(e_{i}\right)=\left\{\tilde{\mathfrak{K}}_{i}\left(e_{i}, s\right), \quad s \in \mathrm{S}\left(e_{i}\right)\right\}
$$

where $\tilde{\mathfrak{K}}_{i}: \mathbb{R} \times[-1,1] \rightarrow \mathbb{R}$ is a continuous (single valued) function.

The proof of Proposition 6 is made iteratively from $i=$ $m$ toward 1 . At each step, we show that $V_{i}$ is positive definite and we look for a positive real number $\ell_{i}$, such that for all $E_{i}$ in $\mathbb{R}^{n-i+1}$

$$
\begin{array}{r}
\max _{s \in \mathrm{S}\left(e_{i}\right)}\left\{\frac{\partial V_{i}}{\partial E_{i}}\left(E_{i}\right)\left(S_{m-i+1} E_{i}+\tilde{\mathfrak{K}}_{i}\left(e_{i}, s\right)\right)\right\} \\
\leq-c_{i} V_{i}\left(E_{i}\right)^{\frac{d_{V}-1}{d_{V}}},
\end{array}
$$

where $c_{i}$ is a positive real number. The Proposition will be proved once we have shown that the former inequality holds for $i=1$.

Step $i=m$ : At this step, $E_{m}=e_{m}$. Note that we have

$$
\begin{aligned}
\max _{s \in \mathrm{S}\left(e_{m}\right)}\left\{\frac{\partial V_{m}}{\partial E_{m}}\left(E_{m}\right) \tilde{\mathfrak{K}}_{m}\left(e_{m}, s\right)\right\} & =-\left|E_{m}\right|^{d_{V}-1} \\
& =-c_{m} V_{m}\left(E_{m}\right)^{\frac{d_{V}-1}{d_{V}}}
\end{aligned}
$$

with $c_{m}=d_{V}^{\frac{d_{V}-1}{d_{V}}}$. Hence, equation (C.1) holds for $i=m$.

Step $i=j$ : Assume $V_{j+1}$ is positive definite and assume $\overline{\text { there exists }}\left(\ell_{j+1}, \ldots, \ell_{m}\right)$ such that (C.1) holds for $j=$ $i-1$. Note that the function $x \mapsto\lfloor x\rceil^{\frac{d_{V}-r_{j}}{r_{j}}}-\left\lfloor e_{i+1}\right\rceil^{\frac{d_{V}-r_{j}}{r_{j+1}}}$ is strictly increasing, is zero iff $x=\left\lfloor e_{j+1}\right\rfloor^{\frac{r_{j}}{r_{j+1}}}$, and therefore has the same sign as $x-\left\lfloor e_{j+1}\right\rceil^{\frac{r_{j}}{r_{j+1}}}$. Thus, for any $e_{j+1}$ fixed in $\mathbb{R}$, the function $\nu \mapsto \bar{V}_{j}\left(\nu, e_{j+1}\right)$ is non negative and is zero only for $v=\left\lfloor e_{j+1}\right\rceil^{\frac{r_{j}}{r_{j+1}}}$. Thus, $\bar{V}_{j}$ is positive and we have

$$
\begin{aligned}
V_{j}\left(E_{j}\right)=0 & \Leftrightarrow\left\{\begin{array}{c}
V_{j+1}\left(E_{j+1}\right)=0 \\
\bar{V}_{j}\left(\ell_{j} e_{j}, e_{j+1}\right)=0
\end{array}\right. \\
& \Leftrightarrow\left\{\begin{array}{l}
E_{j+1}=0 \\
\ell_{j} e_{j}=\left\lfloor e_{j+1}\right)^{\frac{r_{j}}{r_{j+1}}}=0
\end{array}\right.
\end{aligned}
$$

so that $V_{j}$ is positive definite.

On another hand, let $\tilde{V}_{j}\left(\nu, E_{j+1}\right)=V_{j+1}\left(E_{j+1}\right)+$ $\bar{V}_{j}\left(\nu, e_{j+1}\right)$ and let $T_{1}$ be the function defined

$$
T_{1}\left(\nu, E_{j+1}\right)=\max _{s \in \mathrm{S}(\nu)}\left\{\tilde{T}_{1}\left(\nu, E_{j+1}, s\right)\right\}
$$

with $\tilde{T}_{1}$ continuous and defined by

$$
\begin{array}{r}
\tilde{T}_{1}\left(\nu, E_{j+1}, s\right)=\frac{\partial \tilde{V}_{j}}{\partial E_{j+1}}\left(E_{j+1}\right)\left(S_{m-i-1} E_{i+1}+\right. \\
\left.\tilde{\mathfrak{K}}_{j+1}\left(\lfloor\nu\rceil^{\frac{r_{j+1}}{r_{j}}}, s\right)\right)+\frac{c_{j+1}}{2} \tilde{V}_{j}\left(\nu, E_{j+1}\right)^{\frac{d_{V}-1}{d_{V}}} .
\end{array}
$$

Let also $T_{2}$ be the continuous real-valued function defined by

$$
T_{2}\left(v, E_{j+1}\right)=-\frac{\partial \tilde{V}_{j}}{\partial \nu}\left(\nu, E_{i+1}\right)\left(e_{j+1}-\lfloor\nu\rceil^{\frac{r_{j+1}}{r_{j}}}\right) .
$$

Note that $T_{1}$ and $T_{2}$ are homogeneous with weight $r_{j}$ for $\nu$ and $r_{i}$ for $e_{i}$ and degree $d_{V}-1$. Besides, they verify the following two properties :

- for all $E_{j+1}$ in $\mathbb{R}^{m-j}, \nu$ in $\mathbb{R}$

$$
T_{2}\left(\nu, E_{j+1}\right) \geq 0
$$


(since $\left(\lfloor\nu\rceil^{\frac{r_{j+1}}{r_{j}}}-e_{j+1}\right)$ and $\left(\lfloor\nu\rceil^{\frac{d_{V}-r_{j}}{r_{j}}}-\left\lfloor e_{j+1}\right\rceil^{\frac{d_{V}-r_{j}}{r_{j+1}}}\right)$ have the same sign)

- for all $\left(\nu, E_{j+1}\right)$ in $\mathbb{R}^{m-j+1} \backslash\{0\}$, and $s$ in $\mathrm{S}(\nu)$, we have the implication

$$
T_{2}\left(\nu, E_{j+1}\right)=0 \Rightarrow \tilde{T}_{1}\left(\nu, E_{j+1}, s\right)<0
$$

since $T_{2}$ is zero only when $\lfloor\nu\rceil^{\frac{r_{j+1}}{r_{j}}}=e_{j+1}$ and

$$
\begin{aligned}
& \tilde{T}_{1}\left(\left\lfloor e_{j+1}\right\rceil^{\frac{r_{j+1}}{r_{j}}}, E_{j+1}, s\right)= \\
& \quad \frac{\partial V_{j+1}}{\partial E_{j+1}}\left(E_{j+1}\right)\left(S_{n-i} E_{j+1}+\tilde{\mathfrak{K}}_{j+1}\left(e_{j+1}, s\right)\right) \\
& +\frac{c_{j+1}}{2} V_{j+1}\left(E_{j+1}\right)^{\frac{d_{V}-1}{d_{V}}} \leq-\frac{c_{j+1}}{2} V_{j+1}\left(E_{j+1}\right)^{\frac{d_{V}-1}{d_{V}}},
\end{aligned}
$$

where we have employed (C.1) for $i=j-1$.

Using Lemmas 4 in Appendix D, there exists $\ell_{j}$ such that

$$
T_{1}\left(\nu, E_{j+1}\right)-\ell_{j} T_{2}\left(\nu, E_{j+1}\right) \leq 0, \forall\left(\nu, E_{j+1}\right) .
$$

Finally, note that

$$
\begin{aligned}
\max _{s \in \mathrm{S}\left(e_{i}\right)}\{ & \left.\frac{\partial V_{j}}{\partial E_{j}}\left(E_{j}\right)\left(S_{m-j+1} E_{j}+\tilde{\mathfrak{K}}_{j}\left(e_{j}, s\right)\right)\right\}= \\
& T_{1}\left(\ell_{j} e_{j}\right)-\ell_{j} T_{2}\left(\ell_{j} e_{j}, E_{j+1}\right)-\frac{c_{j+1}}{2} V_{j}\left(E_{j}\right)^{\frac{d_{V}-1}{d_{V}}}
\end{aligned}
$$

Hence, (C.1) holds for $i=j$.

\section{Technical lemmas}

Lemma 2 Let $\eta$ be a continuous functions defined on $\mathbb{R}^{n+1}$ and $f$ a continuous function defined on $\mathbb{R}^{n}$. Let $\mathcal{C}$ be a compact subset of $\mathbb{R}^{n}$. Assume that, for all $x$ in $\mathcal{C}$ and $s$ in $\mathrm{S}(f(x))$,

$$
\eta(x, s)<0 .
$$

Then, there exists $\alpha>0$ such that for all $x$ in $\mathcal{C}$ and $s$ in $\mathrm{S}(f(x))$

$$
\eta(x, s)<-\alpha
$$

PROOF. Assume that for all $k>0$, there exists $x_{k}$ in $\mathcal{C}$ and $s_{k}$ in $\mathrm{S}\left(f\left(x_{k}\right)\right) \subset[-1,1]$ such that

$$
0>\eta\left(x_{k}, s_{k}\right) \geq-\frac{1}{k} .
$$

Then, $\eta\left(x_{k}, s_{k}\right)$ tends to 0 when $k$ tends to infinity. Besides, there exists a subsequence $\left(k_{m}\right)$ such that $x_{k_{m}}$ tends to $x^{*}$ in $\mathcal{C}$ and $s_{k_{m}}$ tends to $s^{*}$ in $[-1,1]$. Since $\eta$ is continuous, it follows that $\eta\left(x^{*}, s^{*}\right)=0$ and we will have a contradiction if $s^{*} \in \mathrm{S}\left(f\left(x^{*}\right)\right)$. If $f\left(x^{*}\right)$ is not zero, then by continuity of $f, s^{*}$ is equal to the sign of $f\left(x^{*}\right)$, and otherwise, $s^{*} \in[-1,1]=\mathrm{S}\left(f\left(x^{*}\right)\right)$. Thus, $s^{*} \in \mathrm{S}\left(f\left(x^{*}\right)\right)$ in all cases.

Lemma 3 Let $\eta$ be a function defined on $\mathbb{R}^{n}$ homogeneous with degree $d$ and weight vector $r=\left(r_{1}, \ldots, r_{n}\right)$, and $V$ a positive definite proper function defined on $\mathbb{R}^{n}$ homogeneous of degree $d_{V}$ with same weight vector $r$. Define $\mathcal{C}=V^{-1}(\{1\})$. If there exists $\alpha$ such that for all $x$ in $\mathcal{C}$

$$
\eta(x)<\alpha,
$$

then for all $x$ in $\mathbb{R}^{n} \backslash\{0\}, \eta(x)<\alpha V(x)^{\frac{d}{d_{V}}}$.

PROOF. Let $x$ in $\mathbb{R}^{n} \backslash\{0\}$. We have $\bar{x}=\frac{x_{i}}{V(x)^{\frac{r_{i}}{d_{V}}}}$ in $\mathcal{C}$. Thus $\eta(\bar{x})<\alpha$ and by homogeneity

$$
\frac{1}{V(x)^{\frac{d}{d_{V}}}} \eta(x)<\alpha
$$

which gives the required inequality.

Lemma 4 Let $\eta$ be a homogeneous function of degree d and weight vector $r$ defined on $\mathbb{R}^{n}$ by

$$
\eta(x)=\max _{s \in \mathrm{S}(f(x))} \tilde{\eta}(x, s)
$$

where $\tilde{\eta}$ is a continuous function defined on $\mathbb{R}^{n+1}$ and $f$ a continuous function defined on $\mathbb{R}^{n}$. Consider a continuous function $\gamma$ homogeneous with same degree and weight vector such that, for all $x$ in $\mathbb{R}^{n} \backslash\{0\}$ and $s$ in $\mathrm{S}(f(x))$

$$
\begin{aligned}
& \gamma(x) \geq 0, \\
& \gamma(x)=0 \quad \Rightarrow \quad \tilde{\eta}(x, s)<0 .
\end{aligned}
$$

Then, there exists $k_{0}>0$ such that, for all $x$ in $\mathbb{R}^{n} \backslash\{0\}$,

$$
\eta(x)-k_{0} \gamma(x)<0 \text {. }
$$

PROOF. Define the homogeneous definite positive function $V(x)=\sum_{i=1}^{n}\left|x_{i}\right|^{\frac{d}{r_{i}}}$ and consider the compact set $\mathcal{C}=V^{-1}(\{1\})$. Assume that for all $k>0$, there exists $x_{k}$ in $\mathcal{C}$ and $s_{k}$ in $\mathrm{S}\left(f\left(x_{k}\right)\right)$ such that

$$
\tilde{\eta}\left(x_{k}, s_{k}\right) \geq k \gamma\left(x_{k}\right) \geq 0
$$

$\tilde{\eta}$ is continuous, and thus bounded on the compact set $\mathcal{C} \times[-1,1]$. Therefore, $\gamma\left(x_{k}\right)$ tends to 0 when $k$ tends to infinity. Besides, there exists a subsequence $\left(k_{m}\right)$ such that $x_{k_{m}}$ tends to $x^{*}$ in $\mathcal{C}$ and $s_{k_{m}}$ tends to $s^{*}$ in $[-1,1]$. It follows that $\gamma\left(x^{*}\right)=0$ since $\gamma$ is continuous. But with the same argument as in the proof of Lemma 2, we have $s^{*} \in \mathrm{S}\left(f\left(x^{*}\right)\right)$. It yields that $\tilde{\eta}\left(x^{*}, s^{*}\right)<0$ by assumption and we have a contradiction.

Therefore, there exists $k_{0}$ such that

$$
\tilde{\eta}(x, s)-k_{0} \gamma(x)<0
$$

for all $x$ in $\mathcal{C}$ and all $s$ in $\mathrm{S}(f(x))$. Thus, with Lemma 2 there exists $\alpha>0$ such that

so that

$$
\tilde{\eta}(x, s)-k_{0} \gamma(x) \leq-\alpha
$$

$$
\eta(x)-k_{0} \gamma(x)<0
$$

for any $x$ in $\mathcal{C}$. The result follows applying Lemma 3 . 
Lemma 5 For a positive bounded continuous function $t \mapsto c(t)$ and an absolutely continuous function $t \mapsto \nu(t)$ satisfying

for almost all $t$ such that $\nu(t) \geq c(t)$ then $\dot{\nu}(t) \leq-\nu(t)^{d}$ with $d$ in $] 0,1[$. Then, for all $t$ in $[0, T[$

$$
\nu(t) \leq \max \left\{0, \max \{\nu(0)-c(0), 0\}^{1-d}-t\right\}^{1 /(1-d)}+\sup _{s \in[0, t]} c(s) .
$$

PROOF. This is a direct consequence of the fact that we have for almost all $t$ such that $\max \{\nu(t)-c, 0\})$ is $C^{1}$

$$
\overparen{\max \{\nu(t)-c, 0\}} \leq-\max \{\nu(t)-c, 0\}^{d}
$$

\section{References}

[1] V. Andrieu, L. Praly, and A. Astolfi. Nonlinear output feedback design via domination and generalized weighted homogeneity. IEEE Conference on Decision and Control, 2006.

[2] V. Andrieu, L. Praly, and A. Astolfi. Homogeneous approximation, recursive observer design, and output feedback. SIAM Journal on Control and Optimization, 47(4):1814-1850, 2008.

[3] V. Andrieu, L. Praly, and A. Astolfi. High gain observers with updated gain and homogeneous correction terms. Automatica, 45(2):422-428, 2009.

[4] J.P. Barbot, T. Boukhobza, and M. Djemai. Sliding mode observer for triangular input form. IEEE Conference on Decision and Control, 2:1489 - 1490, 1996.

[5] P. Bernard., L. Praly, and V. Andrieu. Non lipschitz triangular canonical form for uniformly observable controlled systems. IFAC Symposium on Nonlinear Control Systems, 2016.

[6] P. Bernard, L. Praly, and V. Andrieu. On the triangular canonical form for uniformly observable controlled systems. Automatica, 2016, Under review.

[7] G. Besançon. Further results on high gain observers for nonlinear suystems. IEEE Conference on Decision and Control, 3:2904-2909, 1999.

[8] E. Cruz-Zavala, J.A. Moreno, and L. Fridman. Uniform robust exact differentiator. EEE Transactions on Automatic Control, 56(11):2727-2733, 2011.

[9] A. Filippov. Differential equations with discontinuous right-hand sides. Mathematics and its Applications Kluwer Academic Publishers Group, 1988.

[10] J-P. Gauthier and G. Bornard. Observability for any $\mathrm{u}(\mathrm{t})$ of a class of nonlinear systems. IEEE Transactions on Automatic Control, 26:922 - 926, 1981.

[11] J.-P. Gauthier, H. Hammouri, and S. Othman. A simple observer for nonlinear systems application to bioreactors. IEEE Transactions on Automatic Control, 37(6):875-880, 1992.

[12] J-P. Gauthier and I. Kupka. Deterministic observation theory and applications. Cambridge University Press, 2001.
[13] H. K. Khalil and L. Praly. High-gain observers in nonlinear feedback control. Int. J. Robust. Nonlinear Control, 24, April 2013.

[14] A. Levant. Higher-order sliding modes and arbitrary-order exact robust differentiation. Proceedings of the European Control Conference, pages 996-1001, $2001 \mathrm{~b}$.

[15] A. Levant. Higher-order sliding modes, differentiation and output-feedback control. International Journal of Control, 76(9-10):924-941, 2003.

[16] A. Levant. Homogeneity approach to high-order sliding mode design. Automatica, 41(5):823-830, 2005.

[17] J. Moreno and A. Vargas. Approximate high-gain observers for uniformly observable nonlinear systems. In Decision and Control, 2000. Proceedings of the 39th IEEE Conference on, volume 1, pages 784-789. IEEE, 2000.

[18] H. Nakamura, Y. Yamashita, and H. Nishitani. Lyapunov functions for homogeneous differential incolusions. IFAC Symposium on Nonlinear Control Systems, 2004.

[19] F.-A. Ortiz-Ricardez, T. Sanchez, and J.-A. Moreno. Smooth lyapunov function and gain design for a second order differentiator. IEEE Conference on Decision and Control, pages 5402-5407, 2015.

[20] C. Qian. A homogeneous domination approach for global output feedback stabilization of a class of nonlinear systems. Proceedings of the American Control Conference, 2005.

[21] C. Qian and W. Lin. Recursive observer design, homogeneous approximation, and nonsmooth output feedback stabilization of nonlinear systems. IEEE Transactions on Automatic Control, 51(9), 2006.

[22] G. Smirnov. Introduction to the theory of differential inclusions, volume 41. Graduate studies in Mathematics, 2001.

[23] E. Sontag. Smooth stabilization implies coprime factorization. IEEE Transactions on Automatic Control, 34(4), 1989.

[24] E. Sontag and Y. Wang. On characterizations of the input-to-state stability property. Systems \& Control Letter, 24:351-359, 1995.

[25] A. R. Teel. Nonlinear small gain theorem for the analysis of control systems with saturations. IEEE Transactions on Automatic Control, 41(9):1256$1270,1996$.

[26] A. Tornambe. Use of asymptotic observers having high gains in the state and parameter estimation. IEEE Conference on Decision and Control, 2:17911794, 1989.

[27] B. Yang and W. Lin. Homogeneous observers, iterative design, and global stabilization of highorder nonlinear systems by smooth output feedback. IEEE Transactions on Automatic Control, 49(7):1069-1080, 2004. 